\title{
Stress-Induced Phase Transformations in Shape-Memory Polycrystals
}

\author{
KaushiK Bhattacharya \& AnJa Schlömerkemper
}

\author{
Communicated by THE EDITORS
}

\begin{abstract}
Shape-memory alloys undergo a solid-to-solid phase transformation involving a change of crystal structure. We examine model problems in the scalar setting motivated by the situation when this transformation is induced by the application of stress in a polycrystalline material made of numerous grains of the same crystalline solid with varying orientations. We show that the onset of transformation in a granular polycrystal with homogeneous elasticity is in fact predicted accurately by the so-called Sachs bound based on the ansatz of uniform stress. We also present a simple example where the onset of phase transformation is given by the Sachs bound, and the extent of phase transformation is given by the constant strain Taylor bound. Finally we discuss the stress-strain relations of the general problem using Milton-Serkov bounds.
\end{abstract}

\section{Introduction}

Shape-memory alloys often display a phenomenon known as superelasticity or pseudoelasticity wherein strains as large as a few percent suffered on the application of stress are completely recovered on the removal of the applied stress. This is shown schematically in Fig. 1. This phenomenon is observed above a critical temperature, and is the manifestation of a stress-induced solid-to-solid martensitic phase transformation between a high symmetry austenite phase and a low symmetry martensite phase. Above the critical temperature, the austenite phase is the stable phase, but the martensite phase can be stabilized by the application of stress. Thus, one starts with the austenite phase at zero stress. The application of stress initially causes elastic strain of the austenite giving rise to the segment $\mathrm{OA}$ in the figure. At some critical stress, point $\mathrm{A}$ in the figure, the austenite begins to transform to the martensite causing the stress-strain curve to bend. The transformation continues through the plateau till the transformation saturates at B and the martensite begins 


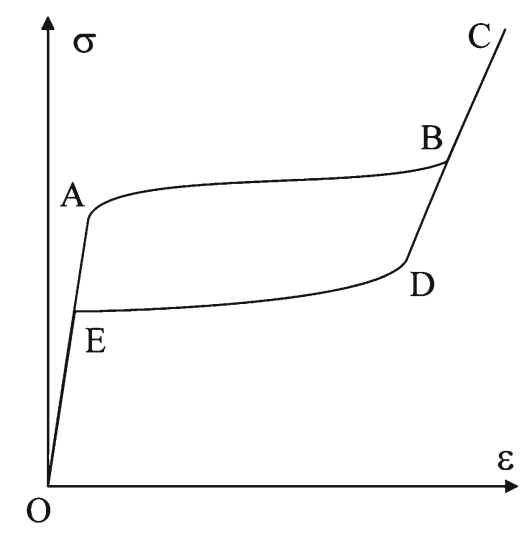

Fig. 1. The phenomenon of superelasticity

to load elastically. Unloading causes the opposite sequence: elastic unloading of the martensite (CD), reverse transformation from martensite to austenite (DE) and elastic unloading of the austenite (EO).

There are at least three notable issues that one has to understand: the stress at which the transformation begins, the strain at which the transformation saturates and the hysteresis. In single crystals, the first two issues are quite well understood (see for example [15,22,24]), and a framework is emerging for the third [25]. However, the understanding of polycrystals lags behind, and motivates this current work. We address the first question - the stress at which transformation begins - and provide some insights towards the second — the strain at which transformation saturates - in a model system.

In a multi-axial setting, the stress at which the transformation begins depends on the direction of the applied stress in stress space, and the locus of the critical stress in the different directions maps out a surface that has been referred to as the transformation yield surface in analogy to plasticity. This is the subject of much recent research, and the various models proposed in the literature have been reviewed recently in [19]. In a single crystal, experimental results are largely consistent with a Schmid-type law that postulates that transformation begins when the applied stress projected along the direction of distortion of any variant of martensite ${ }^{1}$ reaches a critical value. In a polycrystal, however, the different grains are oriented differently and the projections vary by grain. Further, the austenite is not isotropic and thus the elastic moduli and the stresses are not uniform even initially. Furthermore, transformation in one grain can cause incompatibilities with the neighboring grains and thus the transformation is a collective process. Thus the transformation in polycrystalline media is a complex phenomenon. Yet, remarkably, ŠITTNER and NovÁK [24] as well as LEXCELLENT et al. [18] observed that models based on the postulate of uniform stress in the polycrystal reasonably describe the experimental observation

1 This variant should be compatible with the austenite and hence one has to often consider internally twinned habit plane variants. 
of the transformation yield surface. In this paper, we provide some rationale for this in the context of elastic energy minimization and nonlinear homogenization.

We adapt the model of BHATTACHARYA and KoHN [4], who studied the related shape-memory effect, to the current setting of stress-induced martensite. This is introduced in Section 2. We restrict ourselves to the scalar or anti-plane shear setting where the domain is two dimensional and the deformation is scalar. We start with a multiple well energy with one low well for the stable austenite and a number of symmetry-related higher wells for the metastable variants of martensite, and then use relaxation and homogenization methods to understand the effect of a polycrystal. We do so in two settings, an elastic setting where each well is elastic and a constrained setting where each well is restricted to the preferred strain of the variant (that is, the elastic modulus is set to be infinite).

In Section 3 we show rigorously that in the elastic setting and under the assumption that the elastic moduli of all wells are equal and isotropic, the Sachs bound based on the ansatz of uniform stress correctly predicts the onset of the transformation in a granular polycrystal as we announced in [6]. Roughly the idea is the following. We are initially in the austenite and the stress is uniform. As this uniform stress increases, the resolved stress eventually reaches the critical value on one of the variants of martensite in the best oriented grain. At that point, we show, that it is energetically beneficial to create a small nucleus of martensite.

We turn to the constrained setting in the rest of the paper. In Section 4 we present an example of a particular four-variant material and a special checkerboard texture consisting of orientations 0 and $\pi / 4$. We provide a simple argument that reproduces the previous result that the initial yield stress is predicted by the ansatz of constant stress. We then show that the extent of transformation is determined exactly by the ansatz of constant strain. Thus, in this example, the constant stress Sachs lower bound of the energy is optimal for the initiation of transformation, and the constant strain Taylor lower bound is optimal for the saturation of transformation.

We examine general polycrystals in Section 5 using a method proposed by Milton and Serkov [20] that provides bounds on the stress-strain relation. Note that this method is different from common methods that provide bounds on the energy, and bounds on the energy do not translate to a bound on the slope (stress) except possibly at the origin.

Finally, in Section 6 we critically discuss the assumptions and the definitions we make, and the open problems that they leave behind. We also mention possible extensions of this approach.

\section{Model and setting}

We consider anti-plane shear deformations where the domain is in two space dimensions and the displacement is a scalar, $\eta: \mathbb{R}^{2} \rightarrow \mathbb{R}$. We refer to its gradient $e=\nabla \eta \in \mathbb{R}^{2}$ as strain, and this is a vector. We adapt a model of BHATTACHARYA and KoHN [4], and refer to [1,2] for further background on mathematical models of martensitic phase transitions. 


\subsection{Single crystals}

Microscopic energy The microscopic stored energy of a shape-memory alloy single crystal is given by

$$
\psi(e):=\min _{f \in \mathbb{R}^{2}}\left\{\frac{C}{2}|e-f|^{2}+W(f)\right\}, \quad e \in \mathbb{R}^{2},
$$

where $C$ is the elastic modulus assumed to be constant throughout and

$$
W(e):= \begin{cases}0 & \text { if } e=0 \\ w & \text { if } e=e^{(i)}, i=1, \ldots, n \\ \infty & \text { else }\end{cases}
$$

where $e^{(i)}, i=1, \ldots, n$, are the stress-free variants of the martensite and $e=0$ corresponds to the austenite. Note that $\psi$ has a multi-well structure as shown schematically in Fig. 2a with the austenite as the stable phase and the variants of martensite as the metastable phases. We assume self-accommodation, or $0 \in \operatorname{co}\left\{e^{(1)}, \ldots, e^{(n)}\right\}$.

We can obtain the corresponding stress by differentiating the energy density with respect to the strain,

$$
s=\frac{\partial \psi}{\partial e}=C\left(e-e^{(i)}\right), \quad \text { where } e^{(i)}=\operatorname{argmin}_{e^{(j)}, j=1, \ldots, n}\left(e-e^{(j)}\right) .
$$

The stress is a vector in this scalar model. The stress-strain relation is piecewise affine (and discontinuous) with each affine region corresponding to a phase or variant as shown schematically in Fig. $2 b$.

Mesoscopic energy density A material with a multi-well microscopic energy may develop fine-scale microstructure in an attempt to minimize its energy, and therefore it is natural to introduce the mesoscopic energy density. Roughly, the mesoscopic energy density $\hat{\psi}(e)$ is the average energy density of a representative region with an average strain $e$ after it has formed microstructure. Precisely, it is the relaxation of $\psi$ :

$$
\hat{\psi}(e):=\inf _{\eta=e \cdot x \text { on } \partial \Omega}\langle\psi(\nabla \eta(x))\rangle
$$

where $\Omega$ is a domain in physical space $\mathbb{R}^{2}$ and $\langle\cdot\rangle$ denotes the spatial average:

$$
\langle\cdot\rangle:=\frac{1}{|\Omega|} \int_{\Omega} \cdot \mathrm{d} x .
$$

For future use, we define

$$
\hat{W}(e):=\inf _{\eta=e \cdot x \text { on } \partial \Omega}\langle W(\nabla \eta(x))\rangle .
$$

Since we are in the scalar setting, $\hat{\psi}$ is the convexification of $\psi$ (see for example [11]):

$$
\hat{\psi}(e)=\psi^{* *}(e)=\inf _{\langle g\rangle=0}\langle\psi(e+g)\rangle
$$




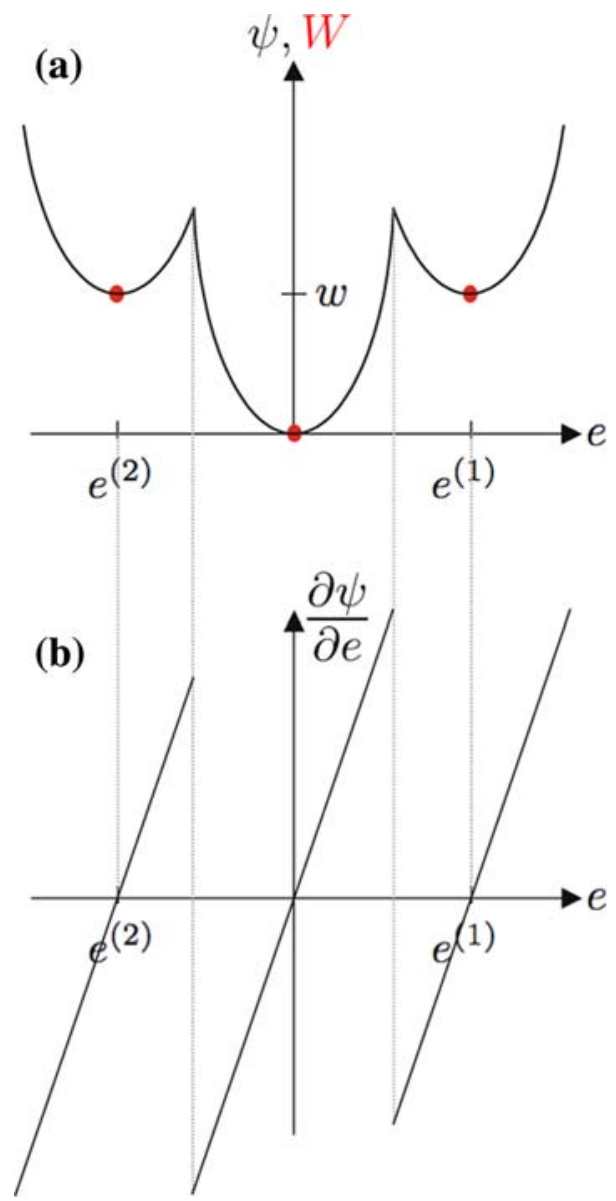

Fig. 2. a A schematic sketch of the microscopic energies $\psi$ and $W$ (the latter is infinite except at $e=0, e^{(1)}$ and $e^{(2)}$ where it takes the values $0, w$ and $w$ respectively as indicated by the three points) with one austenite and two martensitic wells. b The stress-strain relation corresponding to the microscopic energy $\psi$

where

$$
\psi^{*}(s)=\sup _{e \in \mathbb{R}^{2}}\{e \cdot s-\psi(e)\}, \quad s \in \mathbb{R}^{2}
$$

is the Fenchel transform of $\psi$. The Fenchel transform gives the dual or complementary energy density. Similarly, $\hat{W}=W^{* *}$.

The mesoscale energy densities are shown schematically in Fig. $3 \mathrm{a} . \hat{\psi}$ consists of the original wells, and affine regions which are common tangents between the wells. $\hat{W}$ consists of affine regions and is infinite outside a given region. The corresponding dual energies are shown in Fig. 3b. They are very flat in a particular region, and grow away from this region. The stress-strain relations obtained by differentiating the energy densities are shown in Fig. 3c. The stress corresponding to $\hat{\psi}$ 

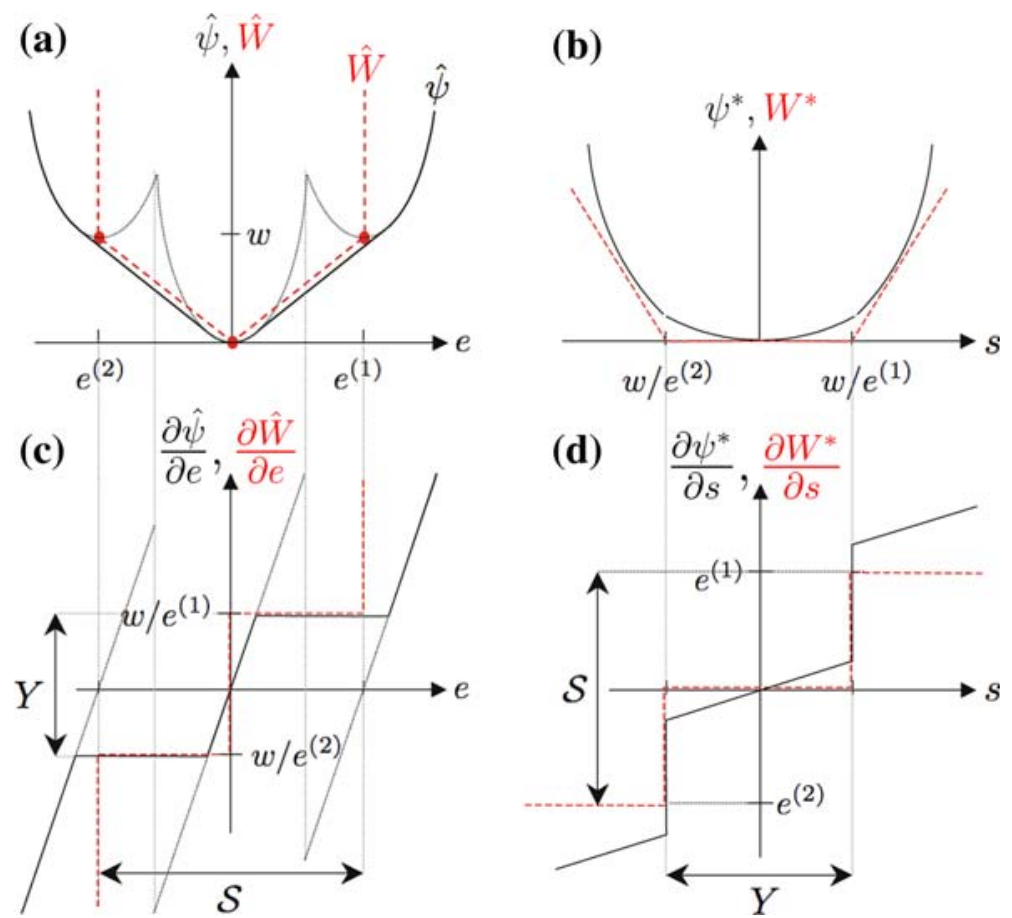

Fig. 3. a The mesoscopic energy densities $\hat{\psi}$ and $\hat{W}$ (dashed) for the microscopic energies shown in Fig. 2a, as well as the corresponding $\mathbf{b}$ dual or complementary energy densities $\psi^{*}$ and $W^{*}$ (dashed), c stress-strain relation obtained by differentiating the energy densities and $\mathbf{d}$ strain-stress relation obtained by differentiating the dual energy densities

consists of affine regions which are connected by regions of constant stress. These regions of constant stress correspond to the common tangents in the energy density, or regions where the material transforms from one phase/variant to another. Thus, these regions mark the boundary of the transformation yield set which is marked $Y$ in the figure. The set of effective transformation strains, marked $\mathcal{S}$, describes the range of strains that can be obtained by transformation. Finally, differentiation of the complementary energy densities gives rise to strain-stress relations, and these are shown in Fig. 3d.

The rest of this subsection makes this schematic picture precise, especially the definition of effective transformation strains and transformation yield set. First, a couple of elementary calculations reveal the relationship between the transforms of $\psi$ and those of $W$. First,

$$
\begin{aligned}
\psi^{*}(s) & =\sup _{e \in \mathbb{R}^{2}} \max _{f \in \mathbb{R}^{2}}\left\{e \cdot s-\frac{C}{2}|e-f|^{2}-W(f)\right\} \\
& =\max _{f \in \mathbb{R}^{2}} \sup _{e \in \mathbb{R}^{2}}\left\{e \cdot s-\frac{C}{2}|e-f|^{2}-W(f)\right\}
\end{aligned}
$$




$$
\begin{aligned}
& =\max _{f \in \mathbb{R}^{2}}\left\{\frac{|s|^{2}}{2 C}+f \cdot s-W(f)\right\} \\
& =\frac{|s|^{2}}{2 C}+W^{*}(s) .
\end{aligned}
$$

So in particular, $\lim _{C \rightarrow \infty} \psi^{*}(s)=W^{*}(s)$. Similarly,

$$
\begin{aligned}
\hat{\psi}(e) & =\inf _{\langle g\rangle=0} \min _{f: \Omega \rightarrow \mathbb{R}^{2}}\left\langle\frac{C}{2}|e+g-f|^{2}+W(f)\right\rangle \\
& =\inf _{\langle g\rangle=0} \min _{\bar{f} \in \mathbb{R}^{2},\langle\tilde{f}\rangle=0}\left\langle\frac{C}{2}|\bar{f}-e|^{2}+\frac{C}{2}|\tilde{f}-g|^{2}+W(\bar{f}+\tilde{f})\right\rangle \\
& =\min _{\bar{f} \in \mathbb{R}^{2},\langle\tilde{f}\rangle=0}\left\langle\frac{C}{2}|\bar{f}-e|^{2}+W(\bar{f}+\tilde{f})\right\rangle \\
& =\min _{\bar{f} \in \mathbb{R}^{2}}\left\{\frac{C}{2}|\bar{f}-e|^{2}+\hat{W}(\bar{f})\right\} .
\end{aligned}
$$

Above, we have set $\bar{f}=\langle f\rangle$ and $\tilde{f}=f-\bar{f}$ and used the fact that

$$
\langle(\bar{f}-e) \cdot(\tilde{f}-g)\rangle=(\bar{f}-e) \cdot\langle\tilde{f}-g\rangle=0
$$

We are now in a position to characterize $\hat{\psi}$ by characterizing $\hat{W}$. We show that

$$
\hat{W}(e)= \begin{cases}w \max _{s_{0} \in \mathbb{S}^{1}} \frac{s_{0} \cdot e}{\max _{i} s_{0} \cdot e^{(i)}} & \text { if } e \in \mathcal{S} \\ \infty & \text { else }\end{cases}
$$

where $\mathcal{S}:=\operatorname{co}\left\{e^{(1)}, \ldots, e^{(n)}\right\}$ is defined to be the set of effective transformation strains of a single crystal and $\mathbb{S}^{1}=\left\{s \in \mathbb{R}^{2}:|s|=1\right\}$. To prove (3), we first note that

$$
\begin{aligned}
W^{*}(s) & =\sup _{e \in \mathbb{R}^{2}}\{s \cdot e-W(e)\} \\
& =\max _{g \in\left\{0, e^{(i)}\right\}}\{s \cdot g-W(g)\} \\
& =\max \left\{0, \max _{i} s \cdot e^{(i)}-w\right\} \\
& = \begin{cases}0 & \text { if } s \in Y, \\
\max _{i} s \cdot e^{(i)}-w & \text { else, }\end{cases}
\end{aligned}
$$

where

$$
Y:=\left\{s \in \mathbb{R}^{2}: s \cdot e^{(i)} \leqq w \forall i\right\}
$$


Hence,

$$
\begin{aligned}
\hat{W}(e) & =\sup _{s \in \mathbb{R}^{2}}\left\{s \cdot e-W^{*}(s)\right\} \\
& =\max \left\{\max _{s \in Y} s \cdot e, \sup _{s \notin Y}\left\{s \cdot e-\max _{i} s \cdot e^{(i)}+w\right\}\right\} .
\end{aligned}
$$

Now let $e \notin \mathcal{S}$. Since $0 \in \mathcal{S}=\operatorname{co}\left\{e^{(1)}, \ldots, e^{(n)}\right\}$, it follows that there exists $s_{0} \in \mathbb{S}^{1}$ such that $s_{0} \cdot\left(e-e^{(i)}\right)>0$ for all $i$. Hence,

$$
\sup _{s \notin Y}\left\{s \cdot e-\left(\max _{i} s \cdot e^{(i)}-w\right)\right\}=\infty
$$

establishing the second line of (3). It remains to study the case $e \in \mathcal{S}$. Again, by the same argument, it follows that for any $s_{0} \in \mathbb{S}^{1}$, there exists $i$ such that $s_{0} \cdot\left(e-e^{(i)}\right) \leqq 0$. Hence,

$$
\begin{aligned}
& \sup _{s \notin Y}\left\{s \cdot e-\left(\max _{i} s \cdot e^{(i)}-w\right)\right\} \\
& =\max _{s_{0} \in \mathbb{S}^{1}} \sup _{\substack{\sigma \geqq 0 \\
\max _{i} \sigma s_{0} \cdot e^{(i)}>w}} \sigma\left(s_{0} \cdot e-\max _{i} s_{0} \cdot e^{(i)}\right)+w \\
& =\max _{s_{0} \in \mathbb{S}^{1}} \sup _{\substack{\sigma \geqq 0 \\
\max _{i} \sigma e^{(i)}>w}} \sigma\left\{\min _{i} s_{0} \cdot\left(e-e^{(i)}\right)\right\}+w \\
& =\max _{s_{0} \in \mathbb{S}^{1}} \frac{w}{\max _{i} s_{0} \cdot e^{(i)}}\left(s_{0} \cdot e-\max _{i} s_{0} \cdot e^{(i)}\right)+w \\
& =\max _{s_{0} \in \mathbb{S}^{1}} w \frac{s_{0} \cdot e}{\max _{i} s_{0} \cdot e^{(i)}} .
\end{aligned}
$$

Above, note that the term in braces on line 2 is negative, and therefore the supremum over non-negative $\sigma$ leads to the highest lower bound of the admissible values. Similarly,

$$
\begin{aligned}
\max _{s \in Y} s \cdot e & =\max _{s_{0} \in \mathbb{S}^{1}} \max _{\substack{\sigma \geqq 0 \\
\max _{i} \sigma s_{0} \cdot e^{(i)} \leqq w}} \sigma s_{0} \cdot e \\
& =\max _{s_{0} \in \mathbb{S}^{1}} w \frac{s_{0} \cdot e}{\max _{i} s_{0} \cdot e^{(i)}} .
\end{aligned}
$$

This gives us the remaining first line of (3).

Note that $\hat{W}$, the convexification of $W$, has a corner at the origin, compare Fig. 3. Thus $W^{*}$ has a non-trivial zero set. As we show next, the set $Y$ in (5) can be interpreted as the transformation yield set of a single crystal. 
Stress-induced transformations Consider a single crystal subjected to dead traction $\sigma=s \cdot v$ on the boundary for some given stress $s$, where $v$ denotes the outer normal to $\partial \Omega$. Then, following JAMES [15], the deformation in the crystal is given by solving the minimization problem:

$$
\inf _{\substack{e: \Omega \rightarrow \mathbb{R}^{2} \\ \text { curl } e=0}} \int_{\Omega}(\psi(e)-s \cdot e) \mathrm{d} x=\int_{\Omega} \inf _{e \in \mathbb{R}^{2}}(\psi(e)-s \cdot e) \mathrm{d} x=-|\Omega| \psi^{*}(s) .
$$

The first equality holds since the integrand is independent of $x$. Looking further at the middle expression, and recalling the definition of $\psi$, it is easy to see that the infimum over $e$ is always attained, and that the minimizer is close to 0 (austenite) for $s$ small enough and close to some $e^{(i)}$ (martensite) for large enough $s$. This transition from the austenite to the martensite is known as stress-induced transformation. Indeed, choosing $s=\sigma s_{0}$ for some fixed $s_{0} \in \mathbb{S}^{1}$ and gradually increasing $\sigma$ from zero, it is clear that this exchange of stability takes place exactly when $\sigma s_{0} \cdot e^{(i)}=w$ for some $i$. Thus the transformation occurs on the surface $\left\{s \in \mathbb{R}^{2}: s \cdot e^{(i)}=w\right.$ for some $\left.i\right\}$. We recognize this as the boundary of the set $Y$ defined in (5). Thus in analogy to plasticity, we call the set $Y$ the transformation yield set of the single crystal. By (5), (4) and (1), respectively, we obtain

$$
\begin{aligned}
Y & =\left\{s \in \mathbb{R}^{2}: s \cdot e^{(i)} \leqq w \forall i\right\}=\left\{s \in \mathbb{R}^{2}: W^{*}(s)=0\right\} \\
& =\left\{s \in \mathbb{R}^{2}: \psi^{*}(s)=\frac{|s|^{2}}{2 C}\right\}=\partial \hat{W}(0),
\end{aligned}
$$

where $\partial \hat{W}(0)$ is the subdifferential of $\hat{W}$ at zero, that is, $\partial \hat{W}(0)=\left\{s \in \mathbb{R}^{2}: s \cdot e \leqq\right.$ $\left.\hat{W}(e) \forall e \in \mathbb{R}^{2}\right\}$. Obviously, $\partial \hat{W}(0) \supseteq Y$. To prove the opposite inequality note that $s \notin Y$ implies that there exists some $i$ such that $s \cdot e^{(i)}>w=\hat{W}\left(e^{(i)}\right)$. Hence $s \notin \partial \hat{W}(0)$ and thus $Y=\partial \hat{W}(0)$ follows. Finally, note that $Y$ is the convex dual of $\mathcal{S}:$

$$
Y=\mathcal{S}^{*}:=\left\{s \in \mathbb{R}^{2}: \hat{W}^{*}(s)=0\right\}=\left\{s \in \mathbb{R}^{2}: \hat{\psi}^{*}(s)=\frac{|s|^{2}}{2 C}\right\},
$$

which follows by (1) since $\hat{W}^{*}(s)=W^{*}(s)$ and $\hat{\psi}^{*}(s)=\psi(s)$ for all $s \in \mathbb{R}^{2}$.

\subsection{Polycrystals}

Macroscopic energy density We are interested in polycrystals that are an agglomeration of a large number of single crystals. It is common to describe the texture, that is, the number, shape and orientation of the grains, with a piecewise constant matrix-valued function $R: \Omega \rightarrow \mathrm{SO}(2)$. Subsets of $\Omega$ on which $R$ is constant are called grains. The microscopic (respectively mesoscopic) energy density of a grain oriented by $R$ is given by $\psi\left(R^{T} e\right)$ (respectively $\hat{\psi}\left(R^{T} e\right)$ ). We obtain the overall behavior by nonlinear homogenization. Recalling that $\hat{\psi}$ is convex and assuming 
that we are in a periodic setting, this overall behavior is given by the macroscopic energy density that is defined as

$$
\bar{\psi}(e):=\inf _{\tilde{e} \in \mathcal{C}_{e}}\left\langle\hat{\psi}\left(R^{T}(x) \tilde{e}\right)\right\rangle
$$

where the averages are taken over a periodic cell, $\Omega_{p}$, and

$$
\mathcal{C}_{e}:=\left\{\tilde{e}: \Omega_{p} \rightarrow \mathbb{R}^{2}:\langle\tilde{e}\rangle=e, \operatorname{curl} \tilde{e}=0\right\}
$$

is the set of all compatible strain fields with average $e$. For a comparison with other definitions of microstructure and homogenization including affine boundary conditions and sequences, we refer to [4, p. 111]. Analogously, we define

$$
\bar{W}(e):=\inf _{\tilde{e} \in \mathcal{C}_{e}}\left\langle\hat{W}\left(R^{T}(x) \tilde{e}\right)\right\rangle
$$

Observe that $\bar{W}$ is infinite for any $e \notin \cup_{x \in \Omega_{p}} \mathcal{S}_{R(x)}$ where $\mathcal{S}_{R}$ denotes the set of transformation strains for a crystal oriented with rotation $R: \mathcal{S}_{R}=\left\{e: R^{T} e \in \mathcal{S}\right\}$. Since $\cup \mathcal{S}_{R}$ is bounded, it follows that $\bar{W}$ is infinite outside of a bounded set

$$
\overline{\mathcal{S}}:=\left\{e \in \mathbb{R}^{2}: \bar{W}(e)<\infty\right\}
$$

which we call the set of effective transformation strains of a polycrystal.

It turns out that the relationship between $\bar{\psi}$ and $\bar{W}$ is more subtle than between $\hat{\psi}$ and $\hat{W}$ in (2) before. Note that $\bar{W}$ requires the transformation strain field to be compatible across all grains. On the other hand, $\bar{\psi}$ requires that the sum of elastic and transformation strain be compatible. Thus, $\bar{\psi}$ allows an interplay between elastic and transformation strains. Consequently, $\bar{\psi}$ is in general smaller than the sum of the elastic energy and $\bar{W}$ :

$$
\bar{\psi}(e) \leqq \inf _{f \in \mathbb{R}^{2}}\left\{\frac{C}{2}|e-f|^{2}+\bar{W}(f)\right\}
$$

To prove this, recall by (2),

$$
\begin{aligned}
\bar{\psi}(e) & =\inf _{\tilde{e} \in \mathcal{C}_{e}}\left\langle\min _{f \in \mathbb{R}^{2}}\left(\frac{C}{2}|f-\tilde{e}|^{2}+\hat{W}\left(R^{T} f\right)\right)\right\rangle \\
& =\inf _{\tilde{e} \in \mathcal{C}_{0}}\left\langle\min _{f \in \mathbb{R}^{2}}\left(\frac{C}{2}|e+\tilde{e}-f|^{2}+\hat{W}\left(R^{T} f\right)\right)\right\rangle \\
& =\inf _{\tilde{e} \in \mathcal{C}_{0}} \inf _{\bar{f} \in \mathbb{R}^{2},\langle\tilde{f}\rangle=0}\left\langle\frac{C}{2}|e+\tilde{e}-(\bar{f}+\tilde{f})|^{2}+\hat{W}\left(R^{T}(\bar{f}+\tilde{f})\right)\right\rangle .
\end{aligned}
$$


Since $\langle(e-\bar{f}) \cdot(\tilde{e}-\tilde{f})\rangle=(e-\bar{f}) \cdot\langle\tilde{e}-\tilde{f}\rangle=0$, we have

$$
\begin{aligned}
\bar{\psi}(e) & =\inf _{\tilde{e} \in \mathcal{C}_{0}} \inf _{\bar{f} \in \mathbb{R}^{2},\langle\tilde{f}\rangle=0}\left\langle\frac{C}{2}\left(|e-\bar{f}|^{2}+|\tilde{e}-\tilde{f}|^{2}\right)+\hat{W}\left(R^{T}(\bar{f}+\tilde{f})\right)\right\rangle \\
& \leqq \inf _{\tilde{e} \in \mathcal{C}_{0}} \inf _{\substack{\bar{f} \in \mathbb{R}^{2} \\
\langle\tilde{f}\rangle=0, \operatorname{curl} \tilde{f}=0}}\left\langle\frac{C}{2}\left(|e-\bar{f}|^{2}+|\tilde{e}-\tilde{f}|^{2}\right)+\hat{W}\left(R^{T}(\bar{f}+\tilde{f})\right)\right\rangle \\
& =\inf _{\bar{f} \in \mathbb{R}^{2}, \tilde{f} \in \mathcal{C}_{0}}\left\langle\frac{C}{2}|e-\bar{f}|^{2}+\hat{W}\left(R^{T}(\bar{f}+\tilde{f})\right)\right\rangle \\
& =\inf _{\bar{f} \in \mathbb{R}^{2}}\left\{\frac{C}{2}|e-\bar{f}|^{2}+\inf _{\tilde{f} \in \mathcal{C}_{0}}\left\langle\hat{W}\left(R^{T}(\bar{f}+\tilde{f})\right)\right\rangle\right\} \\
& =\inf _{\bar{f} \in \mathbb{R}^{2}}\left\{\frac{C}{2}|e-\bar{f}|^{2}+\bar{W}(\bar{f})\right\} .
\end{aligned}
$$

The key point is that the mesoscale transformation strain field $\tilde{f}$ does not have to be compatible in general, that is, it does not have to satisfy curl $\tilde{f}=0$. Therefore the opposite inequality is not generally true.

However, the difference vanishes in the limit of large elastic modulus $C$.

Proposition 1. For any $e \in \mathbb{R}^{2}, \lim _{C \rightarrow \infty} \bar{\psi}(e)=\bar{W}(e)$.

Proof. The proof is similar to one used in [12] to derive a constrained theory of magnetostriction. Set $\hat{W}(f, x):=\hat{W}\left(R^{T}(x) f\right)$ and

$$
\begin{aligned}
\mathcal{F}^{C}(u, f) & :=\left\langle\frac{C}{2}|\nabla u-f|^{2}+\hat{W}(f, x)\right\rangle, \\
\mathcal{F}(u) & :=\langle\hat{W}(\nabla u, x)\rangle, \\
\mathcal{A} & :=\left\{u \in H^{1}\left(\Omega_{p}, \mathbb{R}\right): \nabla u \text { periodic },\langle\nabla u\rangle=e\right\} .
\end{aligned}
$$

Note

$$
\bar{\psi}(e)=\inf _{u \in \mathcal{A}, f \in L^{2}\left(\Omega_{p}, \mathbb{R}^{2}\right)} \mathcal{F}^{C}(u, f), \quad \bar{W}(e)=\inf _{u \in \mathcal{A}} \mathcal{F}(u)
$$

Further, for any given $C$, it is possible to show using the direct method of the calculus of variations that the minimizers $u^{C}, f^{C}$ of $\mathcal{F}^{C}$ exist in $\mathcal{A}, L^{2}$, and $u^{0}$ of $\mathcal{F}$ exists in $\mathcal{A}$. Therefore,

$$
\mathcal{F}^{C}\left(u^{C}, f^{C}\right) \leqq \mathcal{F}^{C}\left(u^{0}, \nabla u^{0}\right)=\mathcal{F}\left(u^{0}\right)=K,
$$

where $K$ is independent of $C$. Thus, since $\left\langle\hat{W}\left(f^{C}, x\right)\right\rangle \geqq 0$,

$$
\begin{aligned}
\left\langle\left|\nabla u^{C}-f^{C}\right|^{2}\right\rangle & \leqq \frac{2 K}{C} \rightarrow 0, \quad \text { or } \quad\left(\nabla u^{C}-f^{C}\right) \rightarrow 0 \text { in } L^{2} \quad \text { as } C \rightarrow \infty, \\
\left\langle\hat{W}\left(f^{C}, x\right)\right\rangle & \leqq K \quad \text { so } \quad f^{C} \stackrel{*}{\rightarrow} \bar{f} \text { in } L^{\infty} \text { as } C \rightarrow \infty
\end{aligned}
$$


for a suitable subsequence. Together, we conclude that $\nabla u^{C} \rightarrow \bar{f}$ in $L^{2}$ and using Sobolev embedding that,

$$
u^{C} \rightarrow \bar{u} \text { in } H^{1}\left(\Omega_{p}, \mathbb{R}\right)
$$

for $\bar{u} \in \mathcal{A}$. We now use these limits to construct a new test function:

$$
\mathcal{F}^{C}\left(u^{C}, f^{C}\right) \leqq \mathcal{F}^{C}(\bar{u}, \nabla \bar{u})=\langle\hat{W}(\bar{f}, x)\rangle
$$

or

$$
\left\langle\frac{C}{2}\left|\nabla u^{C}-f^{C}\right|^{2}\right\rangle \leqq\left\langle\hat{W}(\bar{f}, x)-\hat{W}\left(f^{C}, x\right)\right\rangle \rightarrow 0 \text { as } C \rightarrow \infty
$$

The last limit can be inferred from the convexity of $\hat{W}$ and the non-negativity of the left-hand side. Consequently,

$$
\begin{aligned}
\lim _{C \rightarrow \infty} \mathcal{F}^{C}\left(u^{C}, f^{C}\right) & =\lim _{C \rightarrow \infty}\left\langle\frac{C}{2}\left|\nabla u^{C}-f^{C}\right|^{2}\right\rangle+\lim _{C \rightarrow \infty}\left\langle\hat{W}\left(f^{C}, x\right)\right\rangle \\
& =\lim _{C \rightarrow \infty}\left\langle\hat{W}\left(f^{C}, x\right)\right\rangle \\
& \geqq\langle\hat{W}(\nabla \bar{u}, x)\rangle=\mathcal{F}(\bar{u}) \geqq \mathcal{F}\left(u^{0}\right) .
\end{aligned}
$$

The first inequality follows from the convexity of $\hat{W}$ and weak* limit of $f^{C}$, and the second from the definition of $u^{0}$. This implies the statement.

For future use, we also define the macroscopic dual or complementary energy densities:

$$
\overline{\psi^{*}}(s)=\inf _{\operatorname{div} \tilde{s}=0,\langle\tilde{s}\rangle=s}\left\langle\psi^{*}\left(R^{T} \tilde{s}\right)\right\rangle, \quad \overline{W^{*}}(s)=\inf _{\operatorname{div} \tilde{s}=0,\langle\tilde{s}\rangle=s}\left\langle W^{*}\left(R^{T} \tilde{s}\right)\right\rangle .
$$

We now show that these are exactly equal to the dual of the macroscopic energy densities:

$$
\overline{\psi^{*}}=(\bar{\psi})^{*}, \quad \overline{W^{*}}=(\bar{W})^{*} .
$$

Indeed, to see the first equation in (12), note that

$$
\begin{aligned}
(\bar{\psi})^{*}(s) & =\sup _{e \in \mathbb{R}^{2}}\{e \cdot s-\bar{\psi}(e)\} \\
& =\sup _{e \in \mathbb{R}^{2}} \sup _{\tilde{e} \in \mathcal{C}_{e}}\left\langle\tilde{e} \cdot s-\hat{\psi}\left(R^{T} \tilde{e}\right)\right\rangle \\
& =\sup _{\operatorname{curl} \tilde{e}=0}\left\langle\tilde{e} \cdot s-\hat{\psi}\left(R^{T} \tilde{e}\right)\right\rangle .
\end{aligned}
$$


Now, for any rotation $R$ and $e \in \mathbb{R}^{2}, \hat{\psi}\left(R^{T} e\right)=\sup _{s \in \mathbb{R}^{2}}\left\{e \cdot s-\psi^{*}\left(R^{T} s\right)\right\}$. Substituting this point-wise in the equality above, we obtain

$$
\begin{aligned}
(\bar{\psi})^{*}(s) & =\sup _{\operatorname{curl} \tilde{e}=0}\left\langle\tilde{e} \cdot s-\sup _{\tilde{s} \in \mathbb{R}^{2}}\left\{\tilde{e} \cdot \tilde{s}-\psi^{*}\left(R^{T} \tilde{s}\right)\right\}\right\rangle \\
& \left.=\sup _{\operatorname{curl} \tilde{e}=0 \tilde{s}: \Omega_{p} \rightarrow \mathbb{R}^{2}} \inf _{\left.\tilde{e} \cdot s-\tilde{e} \cdot \tilde{s}+\psi^{*}\left(R^{T} \tilde{s}\right)\right\rangle}\langle\tilde{s})+\psi^{*}\left(R^{T} \tilde{s}\right)\right\rangle \\
& \left.=\sup _{\operatorname{curl} \tilde{e}=0 \tilde{s}: \Omega_{p} \rightarrow \mathbb{R}^{2}} \inf _{\tilde{e}}\left\langle\tilde{e} \cdot(s-\tilde{s})+\psi^{*}\left(R^{T} \tilde{s}\right)\right\rangle\right\} . \\
& =\inf _{\tilde{s}: \Omega_{p} \rightarrow \mathbb{R}^{2}}\left\{\left(\sup _{\operatorname{curl} \tilde{e}=0}\langle\tilde{e} \cdot(s-\tilde{s})\rangle\right)+\langle\right.
\end{aligned}
$$

The penultimate equality follows from the saddle point theorem since the integrand is linear (concave) in $\tilde{e}$ and convex in $\tilde{s}$. Now look at the inner variational problem. We have two cases. First, the case when $\langle\tilde{e} \cdot(s-\tilde{s})\rangle=0$ for all curl-free $\tilde{e}$. Then the inner supremum yields zero. The second case is when $\langle\tilde{e} \cdot(s-\tilde{s})\rangle \neq 0$ for some curl-free $\tilde{e}$. In this case, we see (by constructing a new test field by multiplying with an arbitrary constant) that the supremum is $+\infty$. Thus, when we study the outer variational problem, we see that we may disregard the second case from the allowable $\tilde{s}$. In short, we can restrict ourselves to the first case. Recalling the Helmholtz decomposition of $L^{2}$ into curl-free, divergence-free and constant fields, we see that the first case is equivalent to $\operatorname{div}(s-\tilde{s})=0$ and $\langle s-\tilde{s}\rangle=0$. Thus,

$$
\bar{\psi}^{*}(s)=\inf _{\operatorname{div} \tilde{s}=0,\langle\tilde{s}\rangle=s}\left\langle\psi^{*}\left(R^{T} \tilde{s}\right)\right\rangle=\overline{\psi^{*}}(s)=: \bar{\psi}^{*}(s) .
$$

The second equation in (12) is proved in the same way.

The macroscopic dual energy densities satisfy an inequality analogous to (10). Let $s \in \mathbb{R}^{2}$. Then, by (10),

$$
\begin{aligned}
\bar{\psi}^{*}(s) & =\sup _{e \in \mathbb{R}^{2}}\{s \cdot e-\bar{\psi}(e)\} \\
& \geqq \sup _{e \in \mathbb{R}^{2}}\left\{s \cdot e-\inf _{f \in \mathbb{R}^{2}}\left\{\frac{C}{2}|e-f|^{2}+\bar{W}(f)\right\}\right\} \\
& =\sup _{f \in \mathbb{R}^{2}} \sup _{e \in \mathbb{R}^{2}}\left\{s \cdot e-\left(\frac{C}{2}|e-f|^{2}+\bar{W}(f)\right)\right\} \\
& =\sup _{f \in \mathbb{R}^{2}}\left\{\frac{|s|^{2}}{2 C}+s \cdot f-\bar{W}(f)\right\} \\
& =\frac{|s|^{2}}{2 C}+\sup _{f \in \mathbb{R}^{2}}\{s \cdot e-\bar{W}(f)\} .
\end{aligned}
$$

Hence

$$
\bar{\psi}^{*}(s) \geqq \frac{|s|^{2}}{2 C}+\bar{W}^{*}(s) .
$$


Finally we show in analogy to Proposition 1 that we have equality in the limit of large $C$.

Proposition 2. For any $s \in \mathbb{R}^{2}, \lim _{C \rightarrow \infty} \bar{\psi}^{*}(s)=\bar{W}^{*}(s)$.

Proof. Since the right-hand side of (14) is greater or equal to $\bar{W}^{*}(s)$ for all $s$ and $C$, we immediately have $\lim _{C \rightarrow \infty} \bar{\psi}^{*}(s) \geqq \bar{W}^{*}(s)$ for all $s$. To prove the opposite inequality, we proceed as in Proposition 1, but simpler. Set

$$
\begin{aligned}
\mathcal{G}^{C}(\tilde{s}) & :=\left\langle\frac{|\tilde{s}|^{2}}{2 C}+W^{*}(\tilde{s}, x)\right\rangle, \\
\mathcal{G}(\tilde{s}) & :=\left\langle W^{*}(\tilde{s}, x)\right\rangle,
\end{aligned}
$$

where $W^{*}(\tilde{s}, x):=W^{*}\left(R^{T}(x) \tilde{s}\right)$, and consider the minimum problems of $\mathcal{G}^{C}(\tilde{s})$ and $\mathcal{G}(\tilde{s})$ with respect to the class of admissible functions

$$
\mathcal{D}_{s}:=\left\{\tilde{s} \in L^{2}\left(\Omega_{p}, \mathbb{R}^{2}\right):\langle\tilde{s}\rangle=s, \operatorname{div} \tilde{s}=0\right\} .
$$

By the direct method of the calculus of variation, the minimizers $s^{C}$ of $\mathcal{G}^{C}(\tilde{s})$ and $s^{0}$ of $\mathcal{G}(\tilde{s})$ exist in $\mathcal{D}_{s}$. Furthermore note that, by (11), we then have

$$
\begin{aligned}
\bar{\psi}^{*}(s) & =\min _{\tilde{s} \in \mathcal{D}_{s}} \mathcal{G}^{C}(\tilde{s})=\mathcal{G}^{C}\left(s^{C}\right), \\
\bar{W}^{*}(s) & =\min _{\tilde{s} \in \mathcal{D}_{s}} \mathcal{G}(\tilde{s})=\mathcal{G}\left(s^{0}\right) .
\end{aligned}
$$

Now observe that

$$
\mathcal{G}^{C}\left(s^{C}\right) \leqq \mathcal{G}^{C}\left(s^{0}\right)=\left\langle\frac{\left|s^{0}\right|^{2}}{2 C}\right\rangle+\left\langle W^{*}\left(s^{0}, x\right)\right\rangle=\left\langle\frac{\left|s^{0}\right|^{2}}{2 C}\right\rangle+\mathcal{G}\left(s^{0}\right) .
$$

This implies $\lim _{C \rightarrow \infty} \mathcal{G}^{C}\left(s^{C}\right) \leqq \mathcal{G}\left(s^{0}\right)$ since $s^{0} \in L^{2}\left(\Omega_{p}, \mathbb{R}^{2}\right)$. Hence we obtain $\lim _{C \rightarrow \infty} \bar{\psi}^{*}(s) \leqq \bar{W}^{*}(s)$ for all $s \in \mathbb{R}^{2}$.

Elementary Bounds To get some insights into the nature of the macroscopic energy density, we use constant test functions in the variational and the dual variational principles to obtain the following elementary bounds:

$$
\begin{aligned}
\psi_{S}(e):=\left\langle\psi^{*}\right\rangle^{*}(e)=\left\langle\hat{\psi}^{*}\right\rangle^{*}(e) & \leqq \bar{\psi}(e) \leqq\left\langle\hat{\psi}\left(R^{T}(x) e\right)\right\rangle \equiv\langle\hat{\psi}\rangle(e)=: \psi_{T}(e) \\
W_{S}(e):=\left\langle W^{*}\right\rangle^{*}(e)=\left\langle\hat{W}^{*}\right\rangle^{*}(e) & \leqq \bar{W}(e) \\
& \leqq\left\langle\hat{W}\left(R^{T}(x) e\right)\right\rangle \equiv\langle\hat{W}\rangle(e)=: W_{T}(e)
\end{aligned}
$$

In analogy to plasticity, we call $\psi_{S}, W_{S}$ the Sachs lower bounds and $\psi_{T}, W_{T}$ the Taylor upper bounds on the energy.

Next we define bounds on the set of effective transformation strains as defined in (9), which provides some insight into the saturation of phase transformation. We set

$$
\mathcal{S}_{T}:=\left\{e \in \mathbb{R}^{2}: W_{T}(e)<\infty\right\}, \quad \mathcal{S}_{S}:=\left\{e \in \mathbb{R}^{2}: W_{S}(e)<\infty\right\}
$$


and recall with respect to the latter that $\mathcal{S}_{R}=\left\{e: R^{T} e \in \mathcal{S}\right\}$; furthermore note that $\mathcal{S} \subset \cup_{x \in \Omega} \mathcal{S}_{R(x)}$ which is bounded.

The bounds in (15) translate to inner and outer bounds on the set of effective transformation strains defined in (9):

$$
\mathcal{S}_{T} \subseteq \overline{\mathcal{S}} \subseteq \mathcal{S}_{S}
$$

In general the inclusions are strict as shown in the setting of the shape-memory effect in $[4,7]$. In Section 4 however we provide an example in which the Taylor bound equals the set of effective transformation strains and thus is sharp, compare Proposition 4.

Stress-induced transformations Consider a polycrystal subjected to dead traction $\sigma=s \cdot v$ on the boundary for some given stress $s$, where $v$ denotes the outer normal to $\partial \Omega$. Then, following again JAMES [15], the deformation in the polycrystal is given by solving the minimization problem:

$$
\inf _{\substack{e: \Omega \rightarrow \mathbb{R}^{2} \\ \operatorname{curl} e=0}} \int_{\Omega}(\psi(e, x)-s \cdot e) \mathrm{d} x .
$$

The integrand depends on $x$, and therefore we cannot minimize inside the integral. However,

$$
\begin{aligned}
\inf _{\substack{e: \Omega \rightarrow \mathbb{R}^{2} \\
\text { curl } e=0}} \int_{\Omega}(\psi(e, x)-s \cdot e) \mathrm{d} x & =\inf _{\bar{e} \in \mathbb{R}^{2}}\left\{\inf _{\substack{\langle e\rangle=\bar{e} \\
\operatorname{curl} e=0}} \int_{\Omega} \psi(e, x) \mathrm{d} x-(s \cdot \bar{e})|\Omega|\right\} \\
& =\inf _{\bar{e} \in \mathbb{R}^{2}}(\bar{\psi}(\bar{e})-s \cdot \bar{e})|\Omega| \\
& =-|\Omega| \bar{\psi}^{*}(s) .
\end{aligned}
$$

Since $\bar{W}$ has a corner at the origin and is infinite outside a certain set, we can argue as before that the minimizing strain in the second equality above will be close to 0 for $\sigma$ smaller than a critical stress $\sigma_{\text {crit }}$. As before, we call the latter the transformation yield stress. In a polycrystal, we have two options for defining this critical stress, using $\bar{W}$ or $\bar{\psi}$. We provide two definitions for the transformation yield set of a polycrystal, which are motivated by (7):

$$
\begin{aligned}
\bar{Y}_{C} & =\left\{s \in \mathbb{R}^{2}: \bar{\psi}^{*}(s)=\frac{|s|^{2}}{2 C}\right\}, \\
\bar{Y} & =\left\{s \in \mathbb{R}^{2}: \bar{W}^{*}(s)=0\right\}=\partial \bar{W}(0),
\end{aligned}
$$

where $\partial \bar{W}(0)$ is the subdifferential of $\bar{W}$ at 0 .

For the Taylor bound on the yield set we set analogously

$$
Y_{T}:=\partial W_{T}(0),
$$

and for the Sachs bound on the yield set we define

$$
Y_{S}:=\bigcap_{x \in \Omega_{p}} Y_{R(x)},
$$


where $Y_{R}$ is the yield set of the crystal oriented with rotation $R$ : $Y_{R}=\left\{s: R^{T} s \in Y\right\}$ with $Y$ as in (5). Note that since $W^{*}=0$ on $Y$, it follows that

$$
\left\langle W^{*}\right\rangle=0 \text { on } Y_{S}
$$

Further, if $Y$ has a non-zero interior (that is, if $e^{(i)} \operatorname{span} \mathbb{R}^{2}$ ), then $Y_{S}$ has a non-zero interior and consequently $W_{S}$ has a corner at the origin. Since it is the lower bound and since $\bar{W}(0)=0$ (by observing that the upper bound $W_{T}(0)=0$ ), it follows that $\bar{W}$ also has a corner at the origin.

By (15), we have $Y_{S} \subseteq \bar{Y} \subseteq Y_{T}$. We show in the subsequent section that $Y_{S}$ is sharp in our setting. In the end of Section 5 we relate these energy-based bounds to the Milton-Serkov bounds on the stress-strain curve.

\section{Onset of phase transformation}

We examine the onset of phase transformation in this section for a polycrystal made of a number of nontrivial grains. We define a granular polycrystal to be one where the orientation $R: \Omega_{p} \rightarrow \mathrm{SO}(2)$ may be written as

$$
R=\sum_{i} R_{i} \chi \Omega_{i}
$$

for $R_{i} \in \mathrm{SO}(2)$ and where the grains $\Omega_{i}$ have non-zero Lebesgue measure in $\mathbb{R}^{2}$. For such granular polycrystals, we show that the transformation yield set is equal to the Sachs bound.

Theorem 1. For any granular polycrystal, let $\bar{Y}_{C}, \bar{Y}$ and $Y_{S}$ be the transformation yield sets and Sachs bound defined in (16), (17) and (19), respectively. Then for any $C$

$$
\bar{Y}=\bar{Y}_{C}=Y_{S}
$$

Proof. We begin by showing that $\bar{Y}_{C}=Y_{S}$. The idea behind the proof is simple. Since the elastic modulus is homogeneous across all grains, the stress and strain are uniform as long as the material is in austenite and certainly as long as the applied stress is in the interior of the Sachs set $Y_{S}$. However, as soon as the stress reaches the boundary of the Sachs bound, it becomes favorable for at least one grain to transform. Below we use this idea to construct a test function.

Given any $s \in \mathbb{R}^{2}$, we have from (14)

$$
\bar{\psi}^{*}(s) \geqq \frac{|s|^{2}}{2 C}+\bar{W}^{*}(s) \geqq \frac{|s|^{2}}{2 C}
$$


since $\bar{W}^{*}(s) \geqq 0$ by $(11)$ and the fact that $W^{*}\left(R^{T} \tilde{s}\right) \geqq 0$ for all $\tilde{s}$. Further, from (11), (12) and (1), we have

$$
\begin{aligned}
\bar{\psi}^{*}(s) & \leqq\left\langle\psi^{*}\left(R^{T} s\right)\right\rangle \\
& =\left\langle\frac{|s|^{2}}{2 C}+W^{*}\left(R^{T} s\right)\right\rangle \\
& =\frac{|s|^{2}}{2 C}+\left\langle W^{*}\left(R^{T} s\right)\right\rangle .
\end{aligned}
$$

Now, if $s \in Y_{S}$, then $W^{*}\left(R^{T}(x) s\right)=0$ for each $x$, compare (20), and it follows

$$
\bar{\psi}^{*}(s) \leqq \frac{|s|^{2}}{2 C} .
$$

Combined with (21), we conclude that $\bar{\psi}^{*}(s)=\frac{|s|^{2}}{2 C}$ so that $s \in \bar{Y}_{C}$. Thus, $\bar{Y}_{C} \supseteq Y_{S}$.

To prove the opposite inclusion, consider $s \notin Y_{S}$. By (12), (13) and (2) we obtain

$$
\begin{aligned}
\bar{\psi}^{*}(s) & =\sup _{\operatorname{curl} e=0}\left\langle s \cdot e-\hat{\psi}\left(R^{T} e\right)\right\rangle \\
& =\sup _{\operatorname{curl} e=0}\left\langle s \cdot e-\min _{f \in \mathbb{R}^{2}}\left(\frac{C}{2}|f-e|^{2}+\hat{W}\left(R^{T} f\right)\right)\right\rangle \\
& =\sup _{\operatorname{curl} e=0} \sup _{f: \Omega \rightarrow \mathbb{R}^{2}}\left\langle s \cdot e-\frac{C}{2}|e-f|^{2}-\hat{W}\left(R^{T} f\right)\right\rangle .
\end{aligned}
$$

Thus,

$$
\bar{\psi}^{*}(s) \geqq\left\langle s \cdot e-\frac{C}{2}|e-f|^{2}-\hat{W}\left(R^{T} f\right)\right\rangle
$$

for any curl-free $e$ and any $f$.

Set $e=\frac{s}{C}$, which is curl-free since $s$ is constant. To pick $f$, note that since $s \notin Y_{S}$, there exists an $\widetilde{x} \in \Omega_{p}$ such that $\hat{W}^{*}\left(R^{T}(\widetilde{x}) s\right) \neq 0$. Since $\hat{W}^{*}\left(R^{T}(\widetilde{x}) s\right) \geqq 0$ for all $s$, we have $\hat{W}^{*}\left(R^{T}(\widetilde{x}) s\right)>0$. Hence there exists $\widetilde{f} \in R(\widetilde{x}) \mathcal{S}$ such that $s \cdot \widetilde{f}>\hat{W}\left(R^{T}(\widetilde{x}) \widetilde{f}\right)$; in particular, $\widetilde{f} \neq 0$. Let $\widetilde{\Omega}_{g}$ be the grain to which $\widetilde{x}$ belongs. Then $R(x)=R(\widetilde{x})$ for all $x \in \widetilde{\Omega}_{g}$ and $s \cdot \widetilde{f}>\hat{W}\left(R^{T}(x) \widetilde{f}\right)$ for all $x \in \widetilde{\Omega}_{g}$. Set $f=\lambda \widetilde{f} \chi_{\widetilde{\Omega}_{g}}$ where $\chi_{\widetilde{\Omega}_{g}}$ is the characteristic function of the grain $\widetilde{\Omega}_{g}$ and $\lambda \leqq 1$. Substituting these $f$ and $e$ in the inequality above,

$$
\begin{aligned}
\bar{\psi}^{*}(s) & \geqq \frac{|s|^{2}}{2 C}+\frac{1}{\left|\Omega_{p}\right|} \int_{\Omega_{p}}\left(s \cdot f-\frac{C}{2}|f|^{2}-\hat{W}\left(R^{T} f\right)\right) \mathrm{d} x \\
& =\frac{|s|^{2}}{2 C}+\frac{1}{\left|\Omega_{p}\right|} \int_{\widetilde{\Omega}_{g}}\left(\lambda\left(s \cdot \tilde{f}-\hat{W}\left(R^{T} \tilde{f}\right)\right)-\frac{C \lambda^{2}}{2}|\widetilde{f}|^{2}\right) \mathrm{d} x
\end{aligned}
$$


where we use the fact that $\hat{W}$ is homogeneous of degree one for small $\lambda$. By the properties of $\widetilde{f}$, the term linear in $\lambda$ in the integrand is strictly positive for all $x \in \widetilde{\Omega}_{g}$. Therefore, for small $\lambda$, the integral is strictly positive and we have

$$
\bar{\psi}^{*}(s)>\frac{|s|^{2}}{2 C} .
$$

It follows that $s \notin \bar{Y}_{C}$. Thus, $\bar{Y}_{C} \subseteq Y_{S}$. Combined with the earlier inequality, we conclude that $\bar{Y}_{C}=Y_{S}$.

We now show that $\bar{Y}=Y_{S}$. We have that $\bar{Y} \supseteq Y_{S}$, and it remains to prove the opposite inequality. Consider any $s \notin Y_{S}$. We know that (22) holds for this $s$. Recalling that $\bar{\psi}^{*}$ is the Fenchel transform of $\bar{\psi}$, we conclude that there is an $e \in \mathbb{R}^{2}$ such that

$$
e \cdot s-\bar{\psi}(e)>\frac{|s|^{2}}{2 C} \text {. }
$$

Now take the limit $C \rightarrow \infty$ and recall from Proposition 1 that $\bar{\psi} \rightarrow \bar{W}$. So,

$$
e \cdot s-\bar{W}(e)>0 \text {. }
$$

We therefore conclude that $s \notin \partial \bar{W}(0)=\left\{s \in \mathbb{R}^{2}: f \cdot s-\bar{W}(f) \leqq 0 \forall f \in \mathbb{R}^{2}\right\}$. However, $\bar{Y}=\partial \bar{W}(0)$, and thus $s \notin \bar{Y}$. The result follows.

\section{Four-variant scalar materials with checkerboard texture}

In this section we study a material that has four variants and a special texture, which we call checkerboard texture. We also limit ourselves to the constrained model with infinite elastic modulus. Independently of our result in Theorem 1 we show that, for this material, the Sachs bound is sharp, that is, $Y_{S}=\bar{Y}$. Furthermore, we show that the Taylor bound on the set of effective transformation strains is sharp. That is, the Taylor bound gives a good estimate of the strain at which the transformation saturates.

A four-variant scalar material has four stress-free variants

$$
e^{(1)}=(\alpha, \beta), \quad e^{(2)}=(-\alpha, \beta), \quad e^{(3)}=(-\alpha,-\beta), \quad e^{(4)}=(\alpha,-\beta)
$$

for some $\alpha, \beta>0$. Without loss of generality we will assume in the following that $0<\alpha \leqq \beta$.

By (3) and an elementary calculation we obtain that the mesoscopic energy of four-variant scalar materials is given by

$$
\hat{W}(e)=w \max \left\{\frac{\left|e_{1}\right|}{\alpha}, \frac{\left|e_{2}\right|}{\beta}\right\} \chi_{\left\{\left|e_{1}\right| \leqq \alpha,\left|e_{2}\right| \leqq \beta\right\}}^{-1},
$$

where $e_{1}, e_{2}$ denote the Cartesian components of $e$ and where $\chi^{-1}(\cdot)$ takes the values 1 and $\infty$ and is defined to be $\frac{1}{\chi(\cdot)}$ with $\chi$ being the standard characteristic function that takes the values 1 and 0 , respectively. 


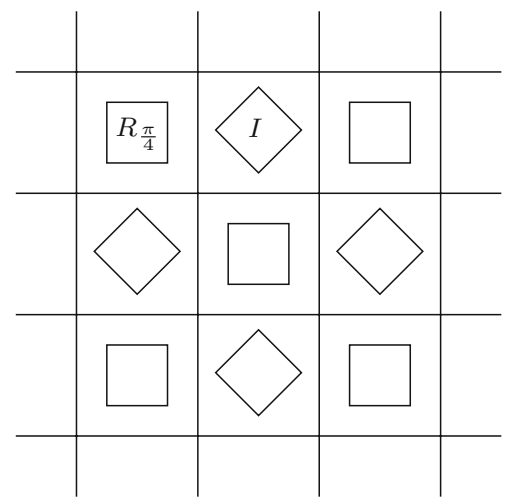

Fig. 4. Sketch of the checkerboard texture and the yield set of each grain

As before, the texture of the material is described by a rotation valued function $R: \Omega \rightarrow \mathrm{SO}(2)$. For brevity we set $R_{\theta}=\left(\begin{array}{cc}\cos \theta & -\sin \theta \\ \sin \theta & \cos \theta\end{array}\right)=:\left(\begin{array}{cc}\mathrm{c} & -\mathrm{s} \\ \mathrm{s} & \mathrm{c}\end{array}\right)$ with $\theta=\theta(x) \in[0,2 \pi]$. A checkerboard texture is defined to have two types of squared grains in the polycrystal, which are equally distributed. One type of grain is described by $R_{0}=I$, the other by the rotation matrix $R_{\pi / 4}$, compare Fig. 4 .

Proposition 3. The yield set of four-variant scalar materials with $\alpha=\beta=1$ and checkerboard texture is

$$
\bar{Y}=Y_{S}=\left\{s=\left(s_{1}, s_{2}\right) \in \mathbb{R}^{2}:\left|s_{1} \pm s_{2}\right| \leqq w,\left|s_{1}\right| \leqq \frac{w}{\sqrt{2}},\left|s_{2}\right| \leqq \frac{w}{\sqrt{2}}\right\} .
$$

Proof. By (19) and (7), $Y_{S}=\bigcap_{x} \mathcal{S}^{*}(x)=\left\{s: \hat{W}^{*}(s)=0\right\} \cap\left\{s: \hat{W}^{*}\left(R_{\frac{\pi}{4}}^{T} s\right)=\right.$ $0\}$. Since

$$
\hat{W}^{*}(s)=\sup _{\left|e_{1}\right| \leqq 1,\left|e_{2}\right| \leqq 1}\left\{\langle e, s\rangle-w \max \left\{\left|e_{1}\right|,\left|e_{2}\right|\right\}\right\}=\max \left\{0,\left|s_{1} \pm s_{2}\right|-w\right\},
$$

we obtain

$$
Y_{S}=\left\{s:\left|s_{1} \pm s_{2}\right| \leqq w,\left|s_{1}\right| \leqq \frac{w}{\sqrt{2}},\left|s_{2}\right| \leqq \frac{w}{\sqrt{2}}\right\}
$$

Since the Sachs bound is an inner estimate of $Y$, we only need to show the opposite inclusion, which we do by choosing appropriate test functions. By (8) we have

$$
\bar{W}(e)=\inf _{\tilde{e} \in \mathcal{C}_{e}}\left\langle\hat{W}\left(R_{\theta}^{T} \tilde{e}\right)\right\rangle \leqq\left\langle\hat{W}\left(R_{\theta}^{T} \tilde{e}\right)\right\rangle .
$$

In particular, consider the vector field $\tilde{e}$ shown in Fig. 5a. The field takes the values indicated by the arrows, and is zero outside the band shown in darker shades of grey. Since it is piecewise constant and satisfies $[|\tilde{e}|] \cdot \hat{t}=0$ at each interface, it is curl-free. Further, for $0<e_{0}<1$

$$
\left\langle\hat{W}\left(R_{\theta}^{T} \tilde{e}\right)\right\rangle=\frac{e_{0} w}{2}\left(\frac{\eta}{\sqrt{2}}+\frac{\eta^{2}}{2}\right) \text { and } e=\langle\tilde{e}\rangle=\frac{e_{0}}{2} \eta(1,0) .
$$




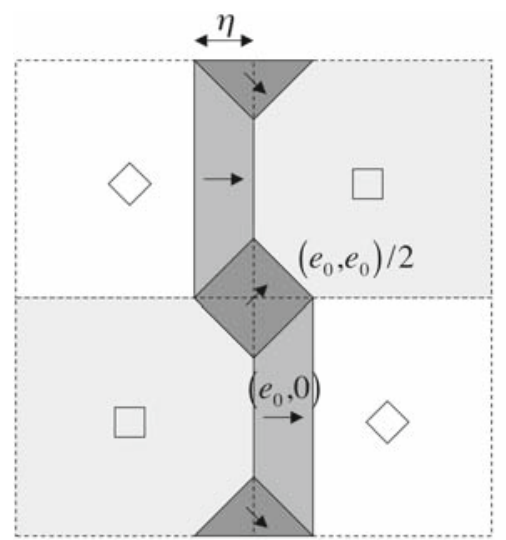

(a)

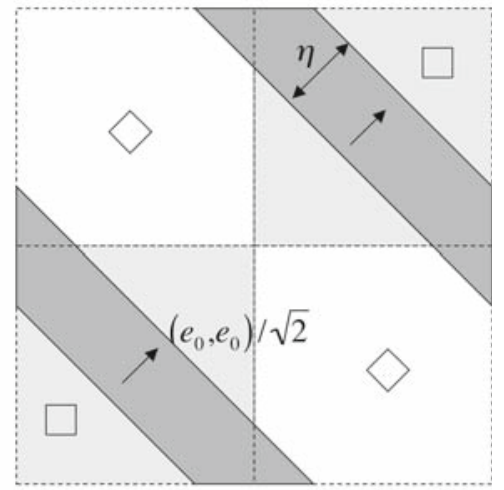

(b)

Fig. 5. Test-fields used to characterize $Y$, compare proof of Proposition 3

It follows that

$$
\bar{W}(e)-\langle s, e\rangle \leqq \frac{e_{0}}{2}\left(\frac{w}{\sqrt{2}}-s_{1}\right) \eta+\frac{e_{0} w}{4} \eta^{2}<0
$$

if and only if $\frac{w}{\sqrt{2}}+\frac{w}{2} \eta<s_{1}$. That is, for $\eta$ small, the inequality holds if $s_{1}>\frac{w}{\sqrt{2}}$. Therefore, $s \notin \bar{Y}$ if $s_{1}>\frac{w}{\sqrt{2}}$, or $\bar{Y} \subseteq\left\{s: s_{1} \leqq \frac{w}{\sqrt{2}}\right\}$. Now consider the vector field shown in Fig. 5b. Following the argument above leads us to the conclusion $\bar{Y} \subseteq\left\{s: s_{1}+s_{2} \leqq w\right\}$. The remaining inclusions are proved analogously.

Our discussion of sharp bounds for the checkerboard is related to an example studied in Bhattacharya and Kohn [4, Section 4.5]. There it is shown that the Taylor bound on the set of recoverable strains of four-variant scalar materials being in their martensitic phase is sharp if the checkerboard texture is given as above ( $R_{0}=I$ and $R_{\frac{\pi}{4}}$ ). Here we prove that this result extends to four-variant scalar materials with checkerboard texture being in their austenitic phase. More precisely, we calculate the set $\mathcal{S}$ of finite macroscopic energy for this material exactly, compare (9). This set can be interpreted as the extent of superelasticity.

To this end we consider the Taylor bound on the macroscopic energy as defined in (15). The properties of $\hat{W}$ asserted in (3) imply that $W_{T}(e)$ is convex and satisfies

$$
W_{T}(e)= \begin{cases}\left\langle\hat{W}\left(R^{T}(x) e\right)\right\rangle & \text { if } e \in \mathcal{S}_{T}, \\ \infty & \text { otherwise }\end{cases}
$$

with $\mathcal{S}_{T}=\bigcap_{x} R(x) \mathcal{S}$, and $\mathcal{S}=\operatorname{co}\left\{e^{(1)}, \ldots, e^{(n)}\right\}$ as before. Moreover, $W_{T}(e)$ is homogeneous of degree one on $\mathcal{T}, W_{T}(0)=0$, and $W_{T}(e) \leqq w$ for all $e \in \mathcal{S}_{T}$.

Proposition 4. For any four-variant scalar material with $\alpha=\beta=1$ and checkerboard texture it holds

$$
\overline{\mathcal{S}}=\mathcal{S}_{T}=\left\{e=\left(e_{1}, e_{2}\right) \in \mathbb{R}^{2}:\left|e_{1}\right| \leqq 1,\left|e_{2}\right| \leqq 1, \frac{\left|e_{1} \pm e_{2}\right|}{\sqrt{2}} \leqq 1\right\}
$$




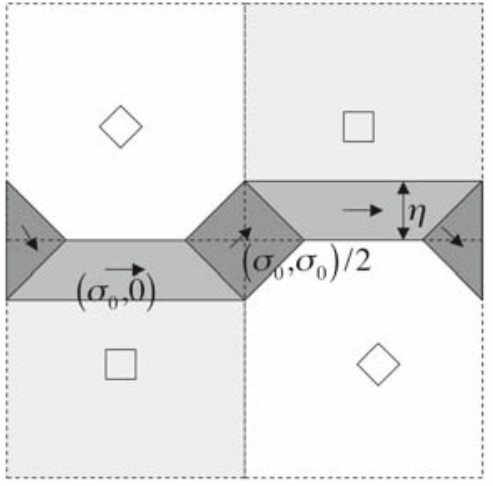

(a)

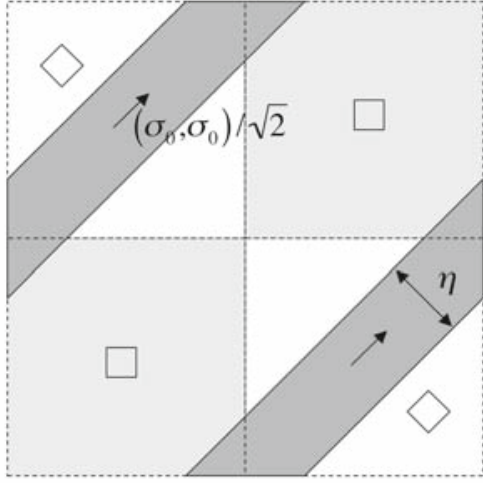

(b)

Fig. 6. Test-fields used to characterize $\overline{\mathcal{S}}$, compare proof of Proposition 4

Proof. By (24)

$$
\mathcal{S}_{T}=\mathcal{S} \cap R_{\frac{\pi}{4}} \mathcal{S}=\left\{e:\left|e_{1}\right| \leqq 1,\left|e_{2}\right| \leqq 1, \frac{\left|e_{1} \pm e_{2}\right|}{\sqrt{2}} \leqq 1\right\} .
$$

This is a subset of $\overline{\mathcal{S}}$ by (15). So we only need to show the opposite inclusion, which we do by choosing appropriate test functions. We obtain

$$
\bar{W}(e) \geqq\left\langle s \cdot e-\hat{W}^{*}\left(R_{\theta}^{T} s\right)\right\rangle
$$

for any field $s$ such that $\operatorname{div} s=0$. Recall that $\hat{W}^{*}\left(\left(\sigma_{0}, 0\right)\right)=\sigma_{0}-w$ if $\sigma_{0}>w$.

Consider the field shown in Fig. 6a. The field takes the values indicated by the arrows, and is zero outside the band shown in darker shades of grey. Since it is piecewise constant and satisfies $[|s|] \cdot \hat{n}=0$ at each interface, it is divergence-free. Further, for $\sigma_{0}>w$, we have from the bound above that

$$
\begin{aligned}
\bar{W}(e) & \geqq \frac{1}{2} \sigma_{0} e_{1} \eta-\frac{1}{2} \eta\left(\sigma_{0}-w\right)-\frac{\eta^{2}}{2}\left(\frac{\sigma_{0}}{\sqrt{2}}-w\right) \\
& =\frac{\eta}{2}\left(e_{1}-1\right) \sigma_{0}+\frac{\eta w}{2}+\frac{\eta^{2}}{2}\left(\frac{\sigma_{0}}{\sqrt{2}}-w\right) .
\end{aligned}
$$

For $\eta$ small enough this tends to infinity as $\sigma_{0} \rightarrow \infty$ if $e_{1}>1$. Thus, $\overline{\mathcal{S}} \subseteq\{e:$ $e_{1} \leqq 1$. We repeat this argument with the field shown in Fig. $6 \mathrm{~b}$ to conclude that $\overline{\mathcal{S}} \subseteq\left\{e: \frac{e_{1}+e_{2}}{\sqrt{2}} \leqq 1\right\}$. The remaining inclusions are proved analogously.

\section{Bounds on stress-strain curves}

In this section we adapt an approach by MiLton and SERKov [20] originally developed to bound the current in nonlinear conduction composites to derive bounds 
on the overall stress-strain relation of a polycrystal. We limit ourselves to the constrained model with infinite elastic modulus.

The main idea of Milton and Serkov's approach is not to bound the energy but to provide bounds on a pair of fields of which each satisfies a differential constraint. Consider $K \subset \mathbb{R}^{4}$ to be the mesoscale stress-strain relation of the single-crystal. In other words, $(e, s) \in K$ if $s$ is the stress associated with a mesoscopic strain $e$. Define,

$$
\begin{aligned}
& \mathcal{W}(e, s):= \begin{cases}0 & \text { if }(e, s) \in K, \\
\infty & \text { else, }\end{cases} \\
& \overline{\mathcal{W}}(\bar{e}, \bar{s}):=\inf _{(e, s) \in \mathcal{A}_{\bar{e}, \bar{s}}}\langle\mathcal{W}(e, s)\rangle,
\end{aligned}
$$

where $\mathcal{A}_{\bar{e}, \bar{s}}:=\left\{(e, s) \in \mathbb{R}^{4}:\langle e\rangle=\bar{e}, \operatorname{curl} e=0,\langle s\rangle=\bar{s}, \operatorname{div} s=0\right\}$. It is possible to show that there exists a set $\bar{K}$ such that

$$
\overline{\mathcal{W}}(e, s)= \begin{cases}0 & \text { if }(e, s) \in \bar{K}, \\ \infty & \text { else },\end{cases}
$$

and that $\bar{K}$ is the macroscopic or effective stress-strain relation.

To obtain bounds on $\bar{K}$, Milton and Serkov use the translation method or the method of compensated compactness. Specifically, if $Q(e, s)$ is quasiconvex (that is, if $Q(\bar{e}, \bar{s}) \leqq\langle Q(e, s)\rangle$ for all $\left.(e, s) \in \mathcal{A}_{\bar{e}, \bar{s}}\right)$, it follows that

$$
\overline{\mathcal{W}}(\bar{e}, \bar{s})-Q(\bar{e}, \bar{s}) \geqq \inf _{(e, s) \in \mathcal{A}_{\bar{e}, \bar{s}}}\langle(\mathcal{W}-Q)(e, s)\rangle \geqq\left\langle(\mathcal{W}-Q)^{*}\right\rangle^{*}(\bar{e}, \bar{s}) .
$$

We choose

$$
Q(e, s)=-\lambda e \cdot s, \quad \lambda>0
$$

and conclude from the form of $\mathcal{W}$ and $\overline{\mathcal{W}}$ and from (25) that

$$
(\bar{e}, \bar{s}) \in \bar{K} \Longrightarrow \lambda \bar{e} \cdot \bar{s} \geqq\left\langle H_{\lambda}^{*}\right\rangle^{*}(\bar{e}, \bar{s}),
$$

where $H_{\lambda}(e, s):=\mathcal{W}(e, s)-Q(e, s)$ and thus

$$
H_{\lambda}^{*}\left(e^{\prime}, s^{\prime}\right)=\sup _{(e, s) \in K}\left(e^{\prime} \cdot s+e \cdot s^{\prime}-\lambda e \cdot s\right) .
$$

If we are only interested in isotropic polycrystals, then we may consider only $\bar{e}=\varepsilon_{0}(1,0)$ and $\bar{s}=\sigma_{0}(1,0)$ for $\varepsilon_{0}, \sigma_{0}>0$. The inequality in (26) implies

$$
\begin{aligned}
\lambda \varepsilon_{0} \sigma_{0} & \geqq \sup _{\left(e^{\prime}, s^{\prime}\right) \in \mathbb{R}^{4}}\left(\varepsilon_{0} s_{1}^{\prime}+\sigma_{0} e_{1}^{\prime}-\left\langle H_{\lambda}^{*}\right\rangle\left(e^{\prime}, s^{\prime}\right)\right) \\
& \geqq \sup _{\left(\varepsilon^{\prime}, \sigma^{\prime}\right) \in \mathbb{R}^{2}}\left(\varepsilon_{0} \sigma^{\prime}+\sigma_{0} \varepsilon^{\prime}-\left\langle H_{\lambda}^{*}\right\rangle\left(\varepsilon^{\prime}(1,0), \sigma^{\prime}(1,0)\right)\right),
\end{aligned}
$$

where we have restricted ourselves to $e^{\prime}, s^{\prime}$ parallel to $(1,0)$ to obtain the second inequality. Therefore, we define

$$
h_{\lambda}^{*}\left(\varepsilon^{\prime}, \sigma^{\prime}\right):=H_{\lambda}^{*}\left(\varepsilon^{\prime}(1,0), \sigma^{\prime}(1,0)\right)
$$




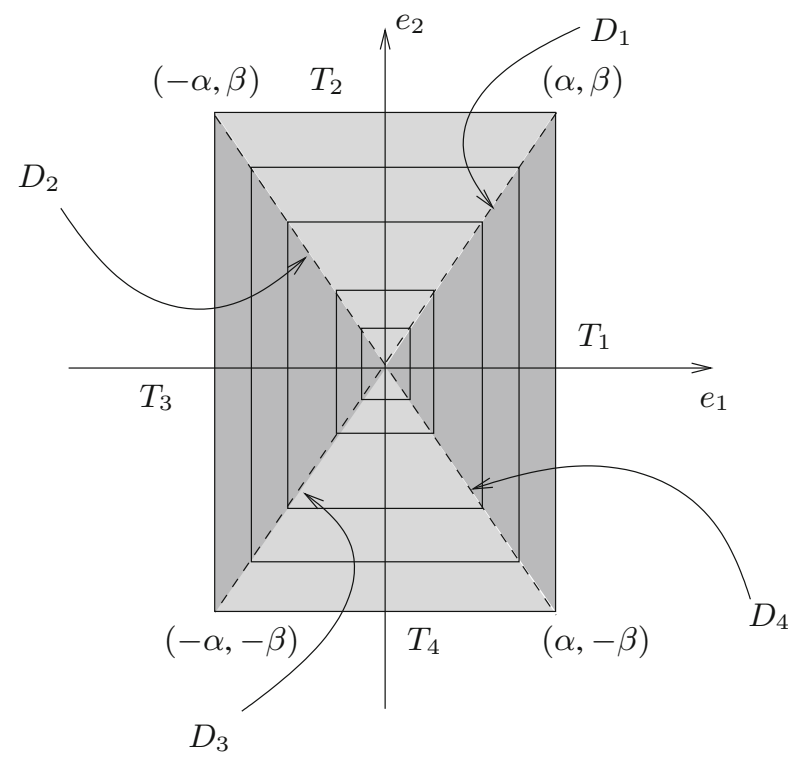

Fig. 7. A sketch of the level sets of $\hat{W}(e)$. Outside the largest rectangle, $\hat{W}(e)=\infty$

and conclude from (26) that

$$
\left(\varepsilon_{0}(1,0), \sigma_{0}(1,0)\right) \in \bar{K} \Longrightarrow \lambda \varepsilon_{0} \sigma_{0} \geqq\left\langle h_{\lambda}^{*}\right\rangle^{*}\left(\varepsilon_{0}, \sigma_{0}\right)
$$

To obtain bounds on $\bar{K}$, we look for pairs $\left(\varepsilon_{0}, \sigma_{0}\right)$ that violate this necessary condition, that is, we search for pairs $\left(\varepsilon_{0}, s_{0}\right)$ that violate the inequality in $(31)$ and obtain $\left(\varepsilon_{0}(1,0), s_{0}(1,0)\right)$ not belonging to $\bar{K}$.

Four-variant material We return to the material with four martensitic variants with transformation strains $( \pm \alpha, \pm \beta)$ considered in the previous section. We recall $\hat{W}$ in (24) and continue to assume that $0<\alpha \leqq \beta$. The level sets of the function $\hat{W}$ are shown in Fig. 7.

Our first task is to characterize the set $K$ for this material. To do so, we recall,

$$
\mathcal{S}=\left\{e \in \mathbb{R}^{2}: \hat{W}(e)<\infty\right\}=\left\{e=\left(e_{1}, e_{2}\right) \in \mathbb{R}^{2}:\left|e_{1}\right| \leqq \alpha,\left|e_{2}\right| \leqq \beta\right\},
$$

and identify

$$
\begin{aligned}
\mathcal{D}_{i} & :=\text { Half diagonals of } \mathcal{S}, \\
\mathcal{T}_{i} & :=\text { Triangles of } \mathcal{S},
\end{aligned}
$$

where $i=1, \ldots, 4$ as shown in Fig. 7. We also recall from (6) and (24) that

$$
\begin{aligned}
Y & =\left\{s \in \mathbb{R}^{2}: e \cdot s \leqq w \max \left\{\frac{\left|e_{1}\right|}{\alpha}, \frac{\left|e_{2}\right|}{\beta}\right\} \forall e \in \mathcal{S}\right\} \\
& =\left\{s=\left(s_{1}, s_{2}\right) \in \mathbb{R}^{2}:\left|\alpha s_{1} \pm \beta s_{2}\right| \leqq w\right\},
\end{aligned}
$$




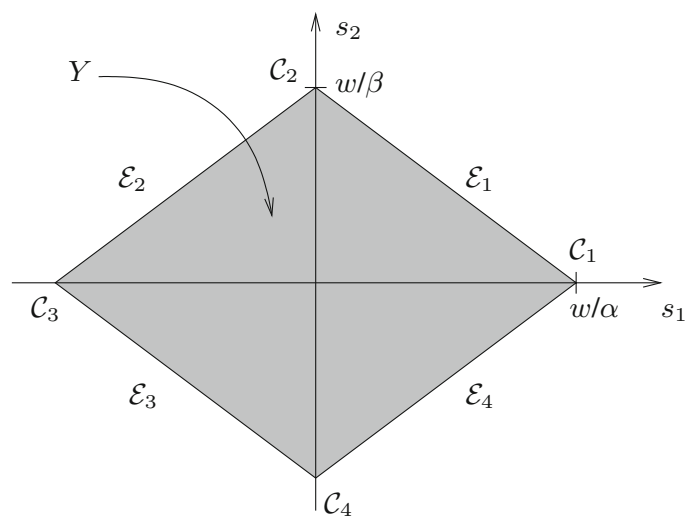

Fig. 8. Sketch of the yield set $Y$

shown in Fig. 8. Define

$$
\begin{aligned}
& \mathcal{C}_{i}=\text { Corner points of } Y, \quad i=1, \ldots, 4, \\
& \mathcal{E}_{i}=\text { Edges of } Y, \quad i=1, \ldots, 4
\end{aligned}
$$

We are now in a position to characterize the set $K$. It follows from $\hat{W}$ in (24) that

$$
K=K_{0} \cup\left(\bigcup_{i=1}^{4} K_{i}\right) \cup B_{i} \cup K_{\infty}
$$

where

$$
\begin{aligned}
& K_{0}:=\{(0, s): s \in Y\}, \\
& K_{i}:=\left\{\left(e, \mathcal{C}_{i}\right): e \in \mathcal{T}_{i}\right\}, \quad i=1, \ldots, 4 \\
& B_{i}:=\left\{(e, s): e \in \mathcal{D}_{i}, s \in \mathcal{E}_{i}\right\}, \\
& K_{\infty}:=\left\{(e, s): e \in \partial \mathcal{S} \text { and } s \text { such that if } e \in \partial \mathcal{S} \cap \partial T_{1}, \text { then } s_{1} \geqq \frac{w}{\alpha}\right. \text { and } \\
& s_{2}=0 ; \text { if } e=(\alpha, \beta), \text { then } s_{1}, s_{2}>0 \text { and } s \in Y^{c} ; \text { analogously for } \\
&\text { other parts of } \partial \mathcal{S}\} .
\end{aligned}
$$

For this $K$, the function $H_{\lambda}^{*}$ defined in (27) is characterized below.

Lemma 1. Assume $s_{1}^{\prime}, s_{2}^{\prime} \geqq 0$. For four-variant scalar materials with the set $K$ given in (33), the function $H_{\lambda}^{*}$ defined in (27) is given by 


$$
\begin{aligned}
& H_{\lambda}^{*}\left(e^{\prime}, s^{\prime}\right) \\
& =\sup _{(e, s) \in \cup_{i=1}^{4} K_{i}}\left(e^{\prime} \cdot s+e \cdot s^{\prime}-\lambda e \cdot s\right) \\
& =\max \left\{\frac{w}{\alpha} e_{1}^{\prime}+\max \left\{0, \alpha s_{1}^{\prime}+\beta s_{2}^{\prime}-\lambda w\right\}, \frac{w}{\beta} e_{2}^{\prime}+\max \left\{0, \alpha s_{1}^{\prime}+\beta s_{2}^{\prime}-\lambda w\right\},\right. \\
& \quad-\frac{w}{\alpha} e_{1}^{\prime}+\max \left\{0,-\alpha s_{1}^{\prime}+\beta s_{2}^{\prime}-\lambda w\right\}, \\
& \left.\quad-\frac{w}{\beta} e_{2}^{\prime}+\max \left\{0, \alpha s_{1}^{\prime}-\beta s_{2}^{\prime}-\lambda w\right\}\right\}
\end{aligned}
$$

if $\left|e_{1}^{\prime}\right| \leqq \lambda \alpha$ and $\left|e_{2}^{\prime}\right| \leqq \lambda \beta$. Otherwise, $H_{\lambda}^{*}\left(e^{\prime}, s^{\prime}\right)$ is infinite.

Proof. Throughout the proof we use again and again that a linear functional over a compact convex subset of $\mathbb{R}^{2}$ attains its maximum at extreme points. On $K_{0}, e=0$ is fixed and we only need to consider the supremum over $s \in Y$. Hence

$$
\begin{aligned}
\sup _{(e, s) \in K_{0}}\left(e^{\prime} \cdot s+e \cdot s^{\prime}-\lambda e \cdot s\right) & =\sup _{s \in Y} e^{\prime} \cdot s=\max _{s \in \mathcal{C}_{i}, i=1, \ldots, 4} e^{\prime} \cdot s \\
& =\max \left\{\frac{w}{\alpha}\left|e_{1}^{\prime}\right|, \frac{w}{\beta}\left|e_{2}^{\prime}\right|\right\} .
\end{aligned}
$$

On $K_{\infty}$ we observe

$$
\begin{aligned}
\sup _{\substack{e \in \partial \mathcal{S} \cap \partial T_{1} \\
s_{1} \geqq \frac{w}{\alpha}, s_{2}=0}}\left(e^{\prime} \cdot s+e \cdot s^{\prime}-\lambda e \cdot s\right) & =\sup _{\substack{e_{2} \in[-\beta, \beta] \\
s_{1} \geqq \frac{w}{\alpha}}}\left(e_{1}^{\prime} s_{1}+\alpha s_{1}^{\prime}+e_{2} s_{2}^{\prime}-\lambda \alpha s_{1}\right) \\
& = \begin{cases}\left.\left(e_{1}^{\prime}-\lambda \alpha\right) \frac{w}{\alpha}+\alpha s_{1}^{\prime}+\beta s_{2}^{\prime}\right) & \text { if } e_{1}^{\prime} \leqq \lambda \alpha, \\
\infty & \text { if } e_{1}^{\prime}>\lambda \alpha .\end{cases}
\end{aligned}
$$

Moreover,

$$
\begin{aligned}
& \sup _{\substack{e=(\alpha, \beta) \\
s_{1}, s_{2}>0, s \in Y^{c}}}\left(e^{\prime} \cdot s+e \cdot s^{\prime}-\lambda e \cdot s\right) \\
= & \sup _{s_{1}, s_{2}>0, s \in Y^{c}}\left(e_{1}^{\prime} s_{1}+e_{2}^{\prime} s_{2}+\alpha s_{1}^{\prime}+\beta s_{2}^{\prime}-\lambda \alpha s_{1}-\lambda \beta s_{2}\right) \\
= & \begin{cases}\sup _{s \in \mathcal{E}_{1}}\left(\left(e_{1}^{\prime}-\lambda \alpha\right) s_{1}+\left(e_{2}^{\prime}-\lambda \beta\right) s_{2}\right)+\alpha s_{1}^{\prime}+\beta s_{2}^{\prime} & \text { if } e_{1}^{\prime} \leqq \lambda \alpha, e_{2}^{\prime} \leqq \lambda \beta, \\
\infty & \text { else }\end{cases} \\
= & \begin{cases}\max \left\{\left(e_{2}^{\prime}-\lambda \beta\right) \frac{w}{\beta}+\alpha s_{1}^{\prime}+\beta s_{2}^{\prime},\right. & \text { if } e_{1}^{\prime} \leqq \alpha, e_{2}^{\prime} \leqq \beta, \\
\infty & \left.\left(e_{1}^{\prime}-\lambda \alpha\right) \frac{w}{\alpha}+\alpha s_{1}^{\prime}+\beta s_{2}^{\prime}\right\}\end{cases}
\end{aligned}
$$

The other cases run analogously. We deduce that the supremum over $K_{\infty}$ is finite on $e^{\prime} \in \lambda \mathcal{S}$ and infinite otherwise.

The set $B_{i}$ does not matter since the corners of $B_{i}$ are considered when calculating the supremum over $K_{i}$, which we do next. 
On $K_{i}, s=\mathcal{C}_{i}$ is fixed and the supremum reduces to a supremum over the corners of $T_{i}$ since it is a linear problem. Thus twelve pairs of points need to be checked: For each $\mathcal{C}_{i}, i=1, \ldots, 4$, there are two corner points of $T_{i}$ in $\partial \mathcal{S}$ as well as the origin. Since $s=\left(\frac{w}{\alpha}, 0\right)$ in $K_{1}$ and since $T_{1}$ has corner points $(0,0),(\alpha,-\beta)$ and $(\alpha, \beta)$, we obtain

$$
\begin{aligned}
& \sup _{(e, s) \in K_{1}}\left(e^{\prime} \cdot s+e \cdot s^{\prime}-\lambda e \cdot s\right) \\
& =\frac{w}{\alpha} e_{1}^{\prime}+\max \left\{0, \alpha s_{1}^{\prime}-\beta s_{2}^{\prime}-\lambda w, \alpha s_{1}^{\prime}+\beta s_{2}^{\prime}-\lambda w\right\} .
\end{aligned}
$$

Similarly for the suprema over $K_{2}, K_{3}$ and $K_{4}$, respectively. Comparing this with the above estimates, we obtain for $s_{1}^{\prime}, s_{2}^{\prime} \geqq 0$ and $\left|e_{1}^{\prime}\right| \leqq \lambda \alpha,\left|e_{2}^{\prime}\right| \leqq \lambda \beta$ the stated formulas for $H_{\lambda}^{*}\left(e^{\prime}, s^{\prime}\right)$.

This is what we know with respect to single crystals. Next we consider polycrystals made of numerous variously oriented grains. For a grain with orientation $\theta$ with respect to a reference single crystal, the stress-strain relation is described by $K_{\theta}=\left\{(e, s):\left(R_{\theta}^{T} e, R_{\theta}^{T} s\right) \in K\right\}$. In analogy to (27) we consider $H_{\lambda, \theta}^{*}\left(e^{\prime}, s^{\prime}\right):=$ $\sup _{(e, s) \in K_{\theta}}\left(e^{\prime} \cdot s+e \cdot s^{\prime}-\lambda e \cdot s\right)$, which becomes, by the definition of $K_{\theta}$,

$$
\begin{aligned}
H_{\lambda, \theta}^{*}\left(e^{\prime}, s^{\prime}\right) & =\sup _{\left(R_{\theta}^{T} e, R_{\theta}^{T} s\right) \in K}\left(e^{\prime} \cdot s+e \cdot s^{\prime}-\lambda e \cdot s\right) \\
& =\sup _{(e, s) \in K}\left(e^{\prime} \cdot R_{\theta} s+R_{\theta} e \cdot s^{\prime}-\lambda R_{\theta} e \cdot R_{\theta} s\right) \\
& =\sup _{(e, s) \in K}\left(R_{\theta}^{T} e^{\prime} \cdot s+e \cdot R_{\theta}^{T} s^{\prime}-\lambda e \cdot s\right)=H_{\lambda}^{*}\left(R_{\theta}^{T} e^{\prime}, R_{\theta}^{T} s^{\prime}\right) .
\end{aligned}
$$

Equiaxed texture We specialize to a polycrystal with an equiaxed texture, in other words, one in which the orientation $\theta$ is equidistributed in $[0,2 \pi)$. Thus, spatial averaging is replaced by orientation averaging. Further, by symmetry of the material and the texture we only need to consider $\theta \in\left[0, \frac{\pi}{2}\right)$. Specializing to this equiaxed situation, the average of the function $h_{\lambda, \theta}^{*}$ defined analogously to (30) is given by

$$
\begin{aligned}
\left\langle h_{\lambda, \theta}^{*}\right\rangle\left(\varepsilon^{\prime}, \sigma^{\prime}\right) & =\left\langle H_{\lambda, \theta}^{*}\left(\varepsilon^{\prime}(1,0), \sigma^{\prime}(1,0)\right)\right\rangle \\
& =\frac{2}{\pi} \int_{0}^{\frac{\pi}{2}} H_{\lambda}^{*}\left(\varepsilon^{\prime}(\cos \theta, \sin \theta), \sigma^{\prime}(\cos \theta, \sin \theta)\right) \mathrm{d} \theta .
\end{aligned}
$$

The following lemma characterizes its Fenchel transform.

Lemma 2. For equiaxed four-variant scalar materials and for $\varepsilon_{0}, \sigma_{0} \geqq 0$, the Fenchel transform of the average of the function $h_{\lambda, \theta}^{*}$ defined analogously to (30) is given by

$$
\left\langle h_{\lambda, \theta}^{*}\right\rangle^{*}\left(\varepsilon_{0}, \sigma_{0}\right)=\max \left\{0, \lambda \alpha\left(\sigma_{0}-\frac{2 w}{\pi} \sqrt{\frac{1}{\alpha^{2}}+\frac{1}{\beta^{2}}}\right)\right\}+\left(g_{\lambda}^{*}\right)^{*}\left(\varepsilon_{0}\right),
$$


where $g_{\lambda}^{*}\left(\sigma^{\prime}\right):=\frac{2}{\pi} \int_{0}^{\frac{\pi}{2}} \max \left\{0, \alpha \sigma^{\prime} \cos \theta+\beta \sigma^{\prime} \sin \theta-\lambda w\right\} \mathrm{d} \theta$ and therefore

$$
\left(g_{\lambda}^{*}\right)^{*}\left(\varepsilon_{0}\right)= \begin{cases}\varepsilon_{0} \sigma_{-}^{\prime} & \text { if } 0 \leqq \varepsilon_{0} \leqq \varepsilon_{1} \\ \sup _{\sigma_{-}^{\prime}<\sigma^{\prime}<\sigma_{+}^{\prime}}\left(\varepsilon_{0} \sigma^{\prime}-g_{\lambda}^{*}\left(\sigma^{\prime}\right)\right. & \text { if } \varepsilon_{1}<\varepsilon_{0}<\varepsilon_{2} \\ \varepsilon_{0} \sigma_{+}^{\prime}-g_{\lambda}^{*}\left(\sigma_{+}^{\prime}\right) & \text { if } \varepsilon_{2} \leqq \varepsilon_{0} \leqq \frac{2}{\pi}(\alpha+\beta) \\ +\infty & \text { if } \frac{2}{\pi}(\alpha+\beta)<\varepsilon_{0}\end{cases}
$$

for

$$
\begin{aligned}
\sigma_{-}^{\prime} & :=\frac{\lambda w}{\sqrt{\alpha^{2}+\beta^{2}}}, \\
\sigma_{+}^{\prime} & :=\frac{\lambda w}{\alpha}, \\
\varepsilon_{1} & :=\min \left\{\inf _{\sigma_{-}^{\prime}<\sigma^{\prime}<\sigma_{+}^{\prime}} \frac{g_{\lambda}^{*}\left(\sigma^{\prime}\right)}{\sigma^{\prime}-\sigma_{-}^{\prime}}, \frac{g_{\lambda}^{*}\left(\sigma_{+}^{\prime}\right)}{\sigma_{+}^{\prime}-\sigma_{-}^{\prime}}\right\}, \\
\varepsilon_{2} & :=\max \left\{\sup _{\sigma_{-}^{\prime}<\sigma^{\prime}<\sigma_{+}^{\prime}} \frac{g_{\lambda}^{*}\left(\sigma_{+}^{\prime}\right)-g_{\lambda}^{*}\left(\sigma^{\prime}\right)}{\sigma_{+}^{\prime}-\sigma^{\prime}}, \frac{g_{\lambda}^{*}\left(\sigma_{+}^{\prime}\right)}{\sigma_{+}^{\prime}-\sigma_{-}^{\prime}}\right\} .
\end{aligned}
$$

Proof. Since we need to consider $\theta \in[0, \pi / 2), \cos \theta \geqq 0, \sin \theta \geqq 0$. Recall $H_{\lambda}^{*}$ from Lemma 1 . Observe that $H_{\lambda}^{*}\left(\varepsilon^{\prime}(\cos \theta, \sin \theta), \sigma^{\prime}(\cos \theta, \sin \theta)\right)$ is infinite if $\varepsilon^{\prime} \cos \theta>\lambda \alpha$ or $\varepsilon^{\prime} \sin \theta>\lambda \beta$ for some $\theta$. It follows that $\left\langle h_{\lambda, \theta}^{*}\right\rangle$ is infinite if $\varepsilon^{\prime}>\lambda \alpha$ (and thus is in particular infinite if $\varepsilon^{\prime}>\lambda \beta$ by assumption). It remains to consider $\varepsilon^{\prime} \leqq \lambda \alpha(\leqq \lambda \beta)$. From (35),

$$
\begin{aligned}
\left\langle h_{\lambda, \theta}^{*}\right\rangle\left(\varepsilon^{\prime}, \sigma^{\prime}\right)= & \frac{2 \varepsilon^{\prime} w}{\pi} \int_{0}^{\frac{\pi}{2}} \max \left\{\frac{\cos \theta}{\alpha}, \frac{\sin \theta}{\beta}\right\} \mathrm{d} \theta \\
& +\frac{2}{\pi} \int_{0}^{\frac{\pi}{2}} \max \left\{0, \alpha \sigma^{\prime} \cos \theta+\beta \sigma^{\prime} \sin \theta-\lambda w\right\} \mathrm{d} \theta \\
= & \frac{2 \varepsilon^{\prime} w}{\pi}\left(\frac{1}{\alpha} \int_{0}^{\arctan \frac{\beta}{\alpha}} \cos \theta \mathrm{d} \theta+\frac{1}{\beta} \int_{\arctan \frac{\alpha}{\beta}}^{\frac{\pi}{2}} \sin \theta \mathrm{d} \theta\right) \\
& +\frac{2}{\pi} \int_{0}^{\frac{\pi}{2}} \max \left\{0, \alpha \sigma^{\prime} \cos \theta+\beta \sigma^{\prime} \sin \theta-\lambda w\right\} \mathrm{d} \theta \\
= & \frac{2 \varepsilon^{\prime} w}{\pi}\left(\frac{1}{\alpha} \sin \arctan \frac{\beta}{\alpha}+\frac{1}{\beta} \cos \arctan \frac{\beta}{\alpha}\right)+g_{\lambda}^{*}\left(\sigma^{\prime}\right) \\
= & \frac{2 \varepsilon^{\prime} w}{\pi} \sqrt{\frac{1}{\alpha^{2}}+\frac{1}{\beta^{2}}}+g_{\lambda}^{*}\left(\sigma^{\prime}\right)
\end{aligned}
$$

with $g_{\lambda}^{*}\left(\sigma^{\prime}\right)=\frac{2}{\pi} \int_{0}^{\frac{\pi}{2}} \max \left\{0, \alpha \sigma^{\prime} \cos \theta+\beta \sigma^{\prime} \sin \theta-\lambda w\right\} \mathrm{d} \theta$. 
To take its Fenchel transform, we notice that $\varepsilon^{\prime}$ and $\sigma^{\prime}$ are decoupled in the formula for $\left\langle h_{\lambda, \theta}^{*}\right\rangle$ above. Therefore,

$$
\begin{aligned}
\left\langle h_{\lambda, \theta}^{*}\right\rangle^{*}\left(\varepsilon_{0}, \sigma_{0}\right) & =\sup _{0 \leqq \varepsilon^{\prime} \leqq \lambda \alpha} \varepsilon^{\prime}\left(\sigma_{0}-\frac{2 w}{\pi} \sqrt{\frac{1}{\alpha^{2}}+\frac{1}{\beta^{2}}}\right)+\sup _{\sigma^{\prime} \geqq 0}\left(\varepsilon_{0} \sigma^{\prime}-g_{\lambda}^{*}\left(\sigma^{\prime}\right)\right) \\
& =\max \left\{0, \lambda \alpha\left(\sigma_{0}-\frac{2 w}{\pi} \sqrt{\frac{1}{\alpha^{2}}+\frac{1}{\beta^{2}}}\right)\right\}+\left(g_{\lambda}^{*}\right)^{*}\left(\varepsilon_{0}\right) .
\end{aligned}
$$

It remains to characterize $\left(g_{\lambda}^{*}\right)^{*}$. It is elementary to verify that

$$
\begin{aligned}
& \sigma_{-}^{\prime}=\lambda w\left(\max _{\theta \in\left[0, \frac{\pi}{2}\right]}(\alpha \cos \theta+\beta \sin \theta)\right)^{-1}, \\
& \sigma_{+}^{\prime}=\lambda w\left(\min _{\theta \in\left[0, \frac{\pi}{2}\right]}(\alpha \cos \theta+\beta \sin \theta)\right)^{-1} .
\end{aligned}
$$

Therefore, by the definition of $g_{\lambda}^{*}$, we then have

$$
g_{\lambda}^{*}\left(\sigma^{\prime}\right)= \begin{cases}0 & \text { if } \sigma^{\prime} \leqq \sigma_{-}^{\prime}, \\ \frac{2}{\pi} \int_{0}^{\frac{\pi}{2}} \max \left\{0, \alpha \sigma^{\prime} \cos \theta+\beta \sigma^{\prime} \sin \theta-\lambda w\right\} \mathrm{d} \theta & \text { if } \sigma_{-}^{\prime}<\sigma^{\prime}<\sigma_{+}^{\prime}, \\ \frac{2}{\pi}(\alpha+\beta) \sigma^{\prime}-\lambda w & \text { if } \sigma_{+}^{\prime} \leqq \sigma^{\prime} .\end{cases}
$$

Hence

$$
\begin{aligned}
& \left(g_{\lambda}^{*}\right)^{*}\left(\varepsilon_{0}\right)=\max \left\{\sup _{0 \leqq \sigma^{\prime} \leqq \sigma_{-}^{\prime}} \varepsilon_{0} \sigma^{\prime}, \sup _{\sigma_{-}^{\prime}<\sigma^{\prime}<\sigma_{+}^{\prime}}\left(\varepsilon_{0} \sigma^{\prime}-g_{\lambda}^{*}\left(\sigma^{\prime}\right)\right),\right. \\
& \left.\sup _{\sigma_{+}^{\prime} \leqq \sigma^{\prime}}\left(\varepsilon_{0} \sigma^{\prime}-\left(\frac{2}{\pi}(\alpha+\beta) \sigma^{\prime}-\lambda w\right)\right)\right\} \text {. }
\end{aligned}
$$

This is infinite if $\varepsilon_{0}>\frac{2}{\pi}(\alpha+\beta)$. Now let $\varepsilon_{0} \leqq \frac{2}{\pi}(\alpha+\beta)$ until the end of the proof. Then $\left(g_{\lambda}^{*}\right)^{*}\left(\varepsilon_{0}\right)$ equals $\varepsilon_{0} \sigma_{-}^{\prime}$ if $\varepsilon_{0} \sigma_{-}^{\prime} \geqq \sup _{\sigma_{-}^{\prime}<\sigma^{\prime}<\sigma_{+}^{\prime}}\left(\varepsilon_{0} \sigma^{\prime}-g_{\lambda}^{*}\left(\sigma^{\prime}\right)\right)$ and $\varepsilon_{0} \sigma_{-}^{\prime} \geqq \varepsilon_{0} \sigma_{+}^{\prime}-\left(\frac{2}{\pi}(\alpha+\beta) \sigma_{+}^{\prime}-\lambda w\right)=\varepsilon_{0} \sigma_{+}^{\prime}-g_{\lambda}^{*}\left(\sigma_{+}^{\prime}\right)$. This yields the inequalities $\varepsilon_{0} \leqq \inf _{\sigma_{-}^{\prime}<\sigma^{\prime}<\sigma_{+}^{\prime}} \frac{g_{\lambda}^{*}\left(\sigma^{\prime}\right)}{\sigma^{\prime}-\sigma_{-}^{\prime}}$ and $\varepsilon_{0} \leqq \frac{g_{\lambda}^{*}\left(\sigma_{+}^{\prime}\right)}{\sigma_{+}^{\prime}-\sigma_{-}^{\prime}}$, respectively, and thus the constraint $\varepsilon_{0} \leqq \varepsilon_{1}$. Similarly, $\left(g_{\lambda}^{*}\right)^{*}\left(\varepsilon_{0}\right)=\varepsilon_{0} \sigma_{+}^{\prime}-g_{\lambda}^{*}\left(\sigma_{+}^{\prime}\right)$ if $\varepsilon_{0} \sigma_{+}^{\prime}-$ $g_{\lambda}^{*}\left(\sigma_{+}^{\prime}\right) \geqq \sup _{\sigma_{-}^{\prime}<\sigma^{\prime}<\sigma_{+}^{\prime}}\left(\varepsilon_{0} \sigma^{\prime}-g_{\lambda}^{*}\left(\sigma^{\prime}\right)\right)$ and $\varepsilon_{0} \sigma_{+}^{\prime}-g_{\lambda}^{*}\left(\sigma_{+}^{\prime}\right) \geqq \varepsilon_{0} \sigma_{-}^{\prime}$. Then $\varepsilon_{0} \geqq \sup _{\sigma_{-}^{\prime}<\sigma^{\prime}<\sigma_{+}^{\prime}} \frac{g_{\lambda}^{*}\left(\sigma_{+}^{\prime}\right)-g_{\lambda}^{*}\left(\sigma^{\prime}\right)}{\sigma_{+}^{\prime}-\sigma^{\prime}}$ and $\varepsilon_{0} \geqq \frac{g_{\lambda}^{*}\left(\sigma_{+}^{\prime}\right)}{\sigma_{+}^{\prime}-\sigma_{-}^{\prime}}$, respectively, and thus the constraint $\varepsilon_{0} \geqq \varepsilon_{2}$ is deduced.

We will apply the following remark in the final bounds. The remark can be verified by observing that $g_{\lambda}^{*}$ is convex. 
Remark 1. For the definitions in Lemma 2,

$$
0 \leqq \varepsilon_{1} \leqq \frac{g_{\lambda}^{*}\left(\sigma_{+}^{\prime}\right)}{\sigma_{+}^{\prime}-\sigma_{-}^{\prime}}=\frac{\frac{2}{\pi}(\alpha+\beta) \sigma_{+}^{\prime}-\lambda w}{\sigma_{+}^{\prime}-\sigma_{-}^{\prime}} \leqq \varepsilon_{2} \leqq \frac{2}{\pi}(\alpha+\beta) .
$$

Therefore, for $\varepsilon_{0} \in\left(\varepsilon_{1}, \varepsilon_{2}\right)$,

$$
\left(g_{\lambda}^{*}\right)^{*}\left(\varepsilon_{0}\right) \geqq \begin{cases}\varepsilon_{0} \sigma_{-}^{\prime} & \text { if } \varepsilon_{0} \leqq \frac{g_{\lambda}^{*}\left(\sigma_{+}^{\prime}\right)}{\sigma_{+}^{\prime}-\sigma_{-}^{\prime}}, \\ \varepsilon_{0} \sigma_{+}^{\prime}-g_{\lambda}^{*}\left(\sigma_{+}^{\prime}\right) & \text { if } \varepsilon_{0}>\frac{g_{\lambda}^{*}\left(\sigma_{+}^{\prime}\right)}{\sigma_{+}^{\prime}-\sigma_{-}^{\prime}} .\end{cases}
$$

We can now provide bounds on the effective stress-strain response.

Proposition 5. For equiaxed four-variant scalar materials and the definitions in Lemma 2, the following pairs of $\left(\varepsilon_{0}, \sigma_{0}\right)$ do not satisfy the inequality in (31) and are thus excluded from the effective stress-strain response $\bar{K}$ :

(a) $\varepsilon_{0}=0$ and $\sigma_{0}>\frac{2 w}{\pi} \sqrt{\frac{1}{\alpha^{2}}+\frac{1}{\beta^{2}}}$,

(b) $0<\varepsilon_{0} \leqq \frac{g_{\lambda}^{*}\left(\sigma_{+}^{\prime}\right)}{\sigma_{+}^{\prime}-\sigma_{-}^{\prime}}$ and any one of the following two conditions:

(i) $\sigma_{0}<\frac{\sigma_{-}^{\prime}}{\lambda}$,

(ii) $\varepsilon_{0}<\alpha$ and $\sigma_{0}>\frac{\lambda \frac{2 w}{\pi} \sqrt{\frac{1}{\alpha^{2}}+\frac{1}{\beta^{2}}} \alpha-\varepsilon_{0} \sigma_{-}^{\prime}}{\lambda \alpha-\lambda \varepsilon_{0}}$,

(c) $\frac{g_{\lambda}^{*}\left(\sigma_{+}^{\prime}\right)}{\sigma_{+}^{\prime}-\sigma_{-}^{\prime}}<\varepsilon_{0} \leqq \frac{2}{\pi}(\alpha+\beta)$ and any one of the following two conditions:

(i) $\sigma_{0}<\frac{\varepsilon_{0} \sigma_{+}^{\prime}-g_{\lambda}^{*}\left(\sigma_{+}^{\prime}\right)}{\lambda \varepsilon_{0}}$,

(ii) $\varepsilon_{0}<\alpha$ and $\sigma_{0}>\frac{\lambda \frac{2 w}{\pi} \sqrt{\frac{1}{\alpha^{2}}+\frac{1}{\beta^{2}}} \alpha-\varepsilon_{0} \sigma_{+}^{\prime}+g_{\lambda}^{*}\left(\sigma_{+}^{\prime}\right)}{\lambda \alpha-\lambda \varepsilon_{0}}$,

(d) $\varepsilon_{0}>\frac{2}{\pi}(\alpha+\beta)$.

We observe that all the bounds asserted in Proposition 5 are independent of $\lambda$. Note that, by (b), the upper bound on $\sigma_{0}$ such that the inequality in (31) is not satisfied is $\frac{\sigma_{-}^{\prime}}{\lambda}=\frac{w}{\sqrt{\alpha^{2}+\beta^{2}}}$ also in the limit as $\varepsilon_{0} \rightarrow 0^{+}$. The regions excluded by the bounds above are shown in Fig. 9 for the case $\alpha=\beta=1$.

Proof. (d) If $\varepsilon_{0}>\frac{2}{\pi}(\alpha+\beta)$, we have, by Lemma 2 , that $\left(g_{\lambda}^{*}\right)^{*}\left(\varepsilon_{0}\right)$ and hence the right-hand side of the inequality in (31) are infinite, that is, there is no restriction on $\sigma_{0}$ in order to violate the inequality in (31).

(a) If $\varepsilon_{0}=0$, the inequality in (31) reads $0 \geqq \max \left\{0, \lambda \alpha\left(\sigma_{0}-\frac{2 w}{\pi} \sqrt{\frac{1}{\alpha^{2}}+\frac{1}{\beta^{2}}}\right)\right\}$ and thus is not satisfied if $\sigma_{0}>\frac{2 w}{\pi} \sqrt{\frac{1}{\alpha^{2}}+\frac{1}{\beta^{2}}}$, which gives (a).

To prove (b), let $0<\varepsilon_{0} \leqq \varepsilon_{1}$ at first, which is less or equal than $\frac{g_{\lambda}^{*}\left(\sigma_{+}^{\prime}\right)}{\sigma_{+}^{\prime}-\sigma_{-}^{\prime}}$ by Remark 1, and assume $\sigma_{0} \leqq \frac{2 w}{\pi} \sqrt{\frac{\alpha^{2}}{+} \frac{1}{\beta^{2}}}$. Then, by Lemma 2, the inequality in (31) is equivalent to $\lambda \varepsilon_{0} \sigma_{0} \geqq \sigma_{-}^{\prime} \varepsilon_{0}$, which is not satisfied if $\sigma_{0}<\frac{\sigma_{-}^{\prime}}{\lambda}$. Next we consider $0<\varepsilon_{0} \leqq \varepsilon_{1}$ and $\sigma_{0}>\frac{2 w}{\pi} \sqrt{\frac{1}{\alpha^{2}}+\frac{1}{\beta^{2}}}$. Then Lemma 2 implies that (31) is violated 


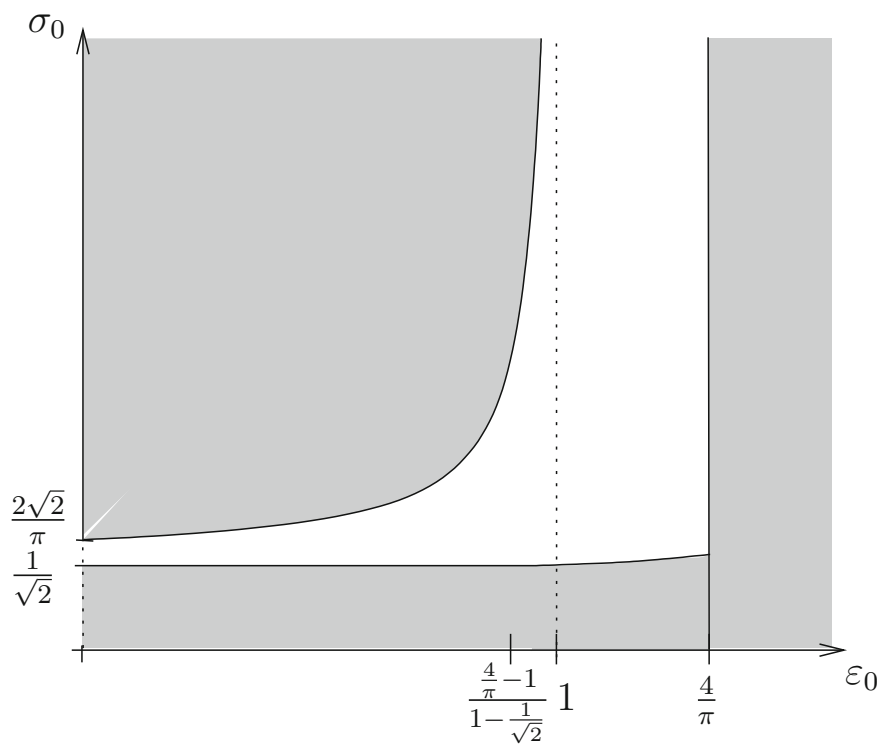

Fig. 9. Sketch of the excluded regions (grey) deduced from (31) for $w=\alpha=\beta=1$. The white regions indicate regions of admissible pairs $\left(\varepsilon_{0}, \sigma_{0}\right)$ for a four-variant scalar material with equiaxed texture. Note that $\frac{g_{\lambda}^{*}\left(\sigma_{+}^{\prime}\right)}{\sigma_{+}^{\prime}-\sigma_{-}^{\prime}}=\frac{\frac{4}{\pi}-1}{1-\frac{1}{\sqrt{2}}}$ by Remark 1

if $\lambda \varepsilon_{0} \sigma_{0}<\lambda \alpha\left(\sigma_{0}-\frac{2 w}{\pi} \sqrt{\frac{1}{\alpha^{2}}+\frac{1}{\beta^{2}}}\right)+\varepsilon_{0} \sigma_{-}^{\prime}$. If $\varepsilon_{0}<\alpha$, this yields the inequality $\sigma_{0}>\frac{\lambda \frac{2 w}{\pi} \sqrt{\frac{1}{\alpha^{2}}+\frac{1}{\beta^{2}}} \alpha-\varepsilon_{0} \sigma_{-}^{\prime}}{\lambda \alpha-\lambda \varepsilon_{0}}$ in (ii). If $\varepsilon_{0}>\alpha$, we obtain $\sigma_{0}<\frac{\varepsilon_{0} \sigma_{-}^{\prime}-\lambda \alpha \frac{2 w}{\pi} \sqrt{\frac{1}{\alpha^{2}}+\frac{1}{\beta^{2}}}}{\lambda \varepsilon_{0}-\lambda \alpha}$. However, the right-hand side of the latter inequality is less than $\frac{\sigma_{-}^{\prime}}{\lambda}$ by an elementary calculation; hence we take the bound from above, which gives (i) for all $\alpha \neq \varepsilon_{0} \leqq \varepsilon_{1}$. If $\varepsilon_{0}=\alpha$, (31) is not satisfied if $0<-\lambda \alpha \frac{2 w}{\pi} \sqrt{\frac{1}{\alpha^{2}}+\frac{1}{\beta^{2}}}+\alpha \sigma_{-}^{\prime}$, which can be shown to be false for all values of the parameters. Hence we do not get any new bound on $\sigma_{0}$ in this case and thus obtain (i) in (b) for all $0<\varepsilon_{0} \leqq \varepsilon_{1}$. To prove this also for $\varepsilon_{1}<\varepsilon_{0} \leqq \frac{g_{\lambda}^{*}\left(\sigma_{+}^{\prime}\right)}{\sigma_{+}^{\prime}-\sigma_{-}^{\prime}}$, we bound $\left(g_{\lambda}^{*}\right)\left(\varepsilon_{0}\right)$ by applying Remark 1 . This then yields the same estimates as before and thus the assertions in (b).

To prove (c), we firstly consider $\varepsilon_{2} \leqq \varepsilon_{0} \leqq \frac{2}{\pi}(\alpha+\beta)$ by observing that $\frac{g_{\lambda}^{*}\left(\sigma_{+}^{\prime}\right)}{\sigma_{+}^{\prime}-\sigma_{-}^{\prime}} \leqq \varepsilon_{2}$ by Remark 1 . In the same lines of arguing as for $(\mathrm{b})$ we obtain the upper bound on $\sigma_{0}$ displayed in (c)(i) if we assume $\sigma_{0} \leqq \frac{2 w}{\pi} \sqrt{\frac{1}{\alpha^{2}}+\frac{1}{\beta^{2}}}$. Next assume that $\sigma_{0}>\frac{2 w}{\pi} \sqrt{\frac{1}{\alpha^{2}}+\frac{1}{\beta^{2}}}$. This yields the lower bound in the case $\varepsilon_{0}<\alpha$. Moreover, in the case $\varepsilon_{0}>\alpha$, the inequality in (31) is violated if $\sigma_{0}<\frac{\varepsilon_{0} \sigma_{+}^{\prime}-g_{\lambda}^{*}\left(\sigma_{+}^{\prime}\right)-\lambda \alpha \frac{2 w}{\pi} \sqrt{\frac{1}{\alpha^{2}}+\frac{1}{\beta^{2}}}}{\lambda \varepsilon_{0}-\lambda \alpha}$. It follows by an elementary calculation that the right-hand side of the latter inequality is less than $\frac{\varepsilon_{0} \sigma_{+}^{\prime}-g_{\lambda}^{*}\left(\sigma_{+}^{\prime}\right)}{\lambda \varepsilon_{0}}$ since $(\alpha+\beta) \sqrt{\frac{1}{\alpha^{2}}+\frac{1}{\beta^{2}}}>\frac{\pi^{2}}{4}$ always. We thus obtain 
the upper bound in (c)(i) for all $\alpha \neq \varepsilon_{0} \geqq \varepsilon_{2}$. If $\varepsilon_{0}=\alpha$, then the inequality in (31) is violated if $0<-\lambda \alpha \frac{2 w}{\pi} \sqrt{\frac{1}{\alpha^{2}}+\frac{1}{\beta^{2}}}+\alpha \sigma_{+}^{\prime}-g_{\lambda}^{*}\left(\sigma_{+}^{\prime}\right)$. The right-hand side of the latter inequality is bounded from above by $-\lambda \alpha \sigma_{0}+\alpha \sigma_{+}^{\prime}-g_{\lambda}^{*}\left(\sigma_{+}^{\prime}\right)$ since here we assume $\sigma_{0}>\frac{2 w}{\pi} \sqrt{\frac{1}{\alpha^{2}}+\frac{1}{\beta^{2}}}$. This however yields the bound displayed in (c)(i), which therefore also holds true for $\varepsilon_{0}=\alpha \geqq \varepsilon_{2}$. Moreover, with (38) we can extend these estimates also to the case $\frac{g_{\lambda}^{*}\left(\sigma_{+}^{\prime}\right)}{\sigma_{+}^{\prime}-\sigma_{-}^{\prime}}<\varepsilon_{0}<\varepsilon_{2}$.

We conclude this section by comparing the bounds in Proposition 5 with the definition of yield set motivated by the elementary bounds on the energy introduced in Section 2. We begin with the yield sets defined in (19) and (18). It is easy to verify for equiaxed four-variant materials that

$$
\begin{aligned}
& Y_{S}=\left\{s \in \mathbb{R}^{2}:|s| \leqq \frac{w}{\sqrt{\alpha^{2}+\beta^{2}}}\right\}, \\
& Y_{T}=\left\{s \in \mathbb{R}^{2}:|s| \leqq \frac{2 w}{\pi} \sqrt{\frac{1}{\alpha^{2}}+\frac{1}{\beta^{2}}}\right\} .
\end{aligned}
$$

The first follows by inserting the formula (32) for the single crystal $Y$ in the definition (19) of $Y_{S}$. To see the second, insert the formula (24) for $\hat{W}$ into the definition (15) of the Taylor bound on the energy to conclude

$$
W_{T}(e)= \begin{cases}w\left\langle\max \left\{\frac{\left|e_{1} \mathrm{c}+e_{2} \mathrm{~s}\right|}{\alpha}, \frac{\left|-e_{1} \mathrm{~s}+e_{2} \mathrm{c}\right|}{\beta}\right\}\right) & \text { if }|e| \leqq \alpha \\ \infty & \text { otherwise }\end{cases}
$$

where $c=\cos \theta, s=\sin \theta$. Since this is isotropic, we evaluate it for $e=(\varepsilon, 0)$ for $0 \leqq \varepsilon \leqq \alpha$ to conclude

$$
\begin{aligned}
W_{T}((\varepsilon, 0)) & =w\left\langle\max \left\{\frac{|\varepsilon \mathrm{c}|}{\alpha}, \frac{|\varepsilon \mathrm{s}|}{\beta}\right\}\right\rangle \\
& =\varepsilon \frac{2 w}{\pi} \int_{0}^{\pi / 2} \max \left\{\frac{\cos \varphi}{\alpha}, \frac{\sin \varphi}{\beta}\right\} \mathrm{d} \varphi \\
& =\varepsilon \frac{2 w}{\pi} \sqrt{\frac{1}{\alpha^{2}}+\frac{1}{\beta^{2}}},
\end{aligned}
$$

where the last equality is obtained as in (36). The result follows by recalling from (18) that $Y_{T}=\partial \bar{W}_{T}(0)$.

Now we compare the yield set motivated by the elementary bounds with those obtained from the Milton-Serkov bounds. Notice from Proposition 5 that for $\varepsilon=0^{+}$, the stress has to lie in the interval $\left[\frac{w}{\sqrt{\alpha^{2}+\beta^{2}}}, \frac{2 w}{\pi} \sqrt{\frac{1}{\alpha^{2}}+\frac{1}{\beta^{2}}}\right]$. In other words, the bounds that we obtain by Milton and Serkov's approach limit the yield set to the range anticipated by the definitions by the elementary Sachs and Taylor bounds. 


\section{Discussion}

This paper addressed issues surrounding the effective behavior of a polycrystal of a shape-memory alloy during stress-induced phase transformation in a model setting of anti-plane shear.

Our first major result shows that the transformation yield set which determines the onset of transformation of a granular polycrystal with homogeneous elastic modulus is predicted exactly by the Sachs bound based on the ansatz of uniform stress (Section 3). An interesting consequence of this fact is the dependence of the transformation yield set on the texture of the polycrystal. The transformation yield set according to the Sachs bound and consequently in any polycrystal considered here depends only on the orientations of the grains (rotations relative to a reference crystal) and is independent of the size, shape and volume fractions, compare also $[5,21]$. The Sachs bound for cubic-to-orthorhombic vectorial materials is given in [21].

We now discuss our assumptions and their implications. The scalar setting implies that relaxation is given by convexification and enables us to use various tools from convex analysis. We conjecture that the same result holds in the vectorial setting in geometrically linear strains and possibly even some models of geometrically nonlinear strain, though this will require a different treatment because relaxation is different from convexification.

The assumption of uniform modulus is essential. Consider a situation where the modulus of the austenite is anisotropic, say cubic as is reasonable in most shape-memory alloys. Then, the modulus differs from grain to grain and thus one has an inhomogeneous elasticity problem at very low stresses. Further, the stresses are likely to be singular at the triple junctions between grains causing the critical stress for transformation to be exceeded locally at infinitesimally small macroscopic stress. Then, the effective stress for the very first transformation event would be zero. This transformation would be extremely localized and would result in a small change to the slope of the stress-strain relation but not a pronounced plateau.

The assumption of granular polycrystal is also essential. In a single crystal, the transformation occurs at constant stress and thus we do not have any hardening in the stress-strain curve shown in Fig. 3. However, this is not necessarily true in a polycrystal. Here, the transformation proceeds gradually with different grains (or regions of grains) transforming at different levels of stress. Further, there can be an interplay between elastic and transformation strains as embodied in the inequality (10). Thus, the stress-strain curve of a polycrystal can display remarkable hardening. Our result characterizes the transformation yield set $Y$ on which transformation begins and creates a deviation from linearity in the stress-strain curve at the transformation yield surface $Y$. However, our result does not quantify the amount of initial transformation or deviation from linearity. It is clear from our argument that this depends on the particular size and shape of grains, and it can be extremely small.

Therefore, it is entirely possible to have a sequence of granular polycrystals (for example, sequentially laminates) with a smaller and smaller deviation from linearity so that the limiting material remains linear beyond our set $Y$ : thus the 


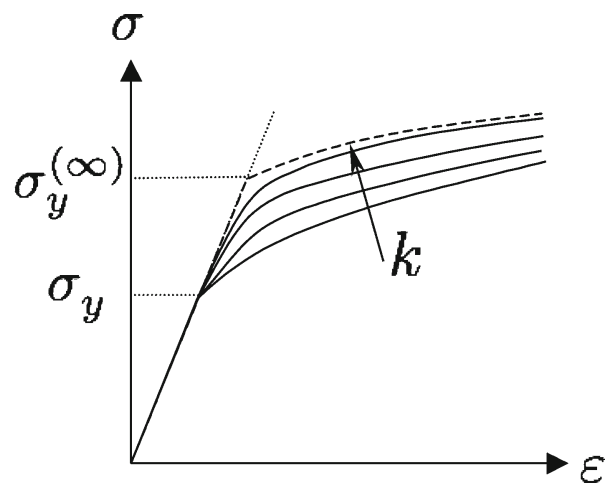

Fig. 10. The transformation yield stress of a sequence of polycrystals, indexed by $k$, each with the same orientations of grains. The transformation yield stress of each element is the same, but the transformation yield stress of the limit (dashed curve) may be different

transformation yield set of the limiting polycrystal would be strictly larger than $Y$. This is illustrated in Fig. 10. Consider a sequence of polycrystals, each with the same range of orientations of grains but with possibly different shapes and sizes. Each polycrystal in the sequence has the same transformation yield stress $\sigma_{y}$. However, the deviation from linearity at $\sigma_{y}$ becomes smaller and smaller, so that the limiting curve is linear at $\sigma_{y}$ and yields at a higher stress $\sigma_{y}^{(\infty)}$. Hence, our result does not hold for polycrystals defined as sequences.

For the above two reasons, the prediction or even the definition of a transformation yield surface is unclear in the general situation. Indeed, the onset of transformation is never completely sharp and the experimental protocol is to use the stress at a selected strain as the transformation yield set [18]. Further, the first cycles of a virgin material are generally observed to be different from the later 'stabilized' cycles. We believe that this is the result of microscopic transformation and analogous plasticity. Finally, one does observe small regions of significantly large local strain before the pronounced formation of the plateau [10].

We note that there are analogous issues in polycrystalline plasticity. There, each grain can slip along a number of slip systems on the application of a critical resolved shear stress, however, the systems differ in orientation from grain to grain. The arguments presented in this paper can easily be adapted to show that a criterion using constant stress is the correct predictor of initial yield under the assumption of isotropic and uniform elastic modulus in anti-plane shear. This has been long recognized by HUTCHINSON [14]. Hutchinson also considered the problem of anisotropic modulus and used a self-consistent method to estimate the local stresses to find that the predicted yield strength is higher than in the isotropic case. This appears to be in deviance with the discussion above until one recognizes that the self-consistent method is a reasonable approximation for the effective stress in the low contrast limit, but has limited information about field fluctuations. More recently, BRENNER et al. [8] have revisited this problem using numerical simulation and the second order method that keeps the field fluctuations; they find that the yield 
stress is indeed lower when one accounts for the fluctuations. Further, it is also true in plasticity that the polycrystal can display hardening even when the single crystal is perfectly plastic.

Our second contribution is a simple example of a particular microstructure where the transformation yield surface is equal to that predicted by the constantstress Sachs bound while the extent of transformation measured by the set of recoverable strains is equal to that predicted by the constant-strain Taylor bound (Section 4). We believe that this curious optimality of bounds is generically suggestive. Our results here and the comparisons with experiments cited earlier show that the constant stress ansatz provides a reasonable indication of the onset of transformation. On the other hand, BHATTACHARYA and KoHN [4] had argued in the context of the shape-memory effect that the constant strain ansatz provides a reasonable indication of the extent of transformation. This was also shown to be consistent with diverse experimental observations [3,23] and numerical simulations [7]. Their idea roughly is that the poorly oriented grains saturate quickly to form a network that prevents further transformation. The picture that emerges then is that transformation begins in isolated well oriented grains and saturates due to the formation of a network of fully-transformed poorly oriented grains. This view is confirmed by the direct experimental observations of BRINSON et al. [9].

A consequence of the fact that the onset and the saturation of transformation are dominated by different grains is that a macroscopic transformation criterion involving critical stress for transformation and the strain of transformation is not going to hold. In a single crystal, recall that the transformation begins when the applied stress $s$ satisfies $s \cdot e^{(i)}=w$ for some $i$. This is the widely used resolved stress criterion that the transformation begins when the component of applied stress along a transformation strain reaches a critical value. In analogy, one would conjecture that in a polycrystal the criterion for transformation is $s \cdot \bar{e}=\bar{w}$ for some macroscopic transformation strain $\bar{e}$ and some constant $\bar{w}$. However, the macroscopic transformation strain is determined by the worst oriented grains. Therefore, a criterion like the one conjectured above would at best describe the stress where the transformation saturates. In contrast, the transformation begins in the best oriented grains and the transformation strain associated with these grains is going to be significantly larger than macroscopic transformation strains. It follows that the resolved stress criterion that seeks to relate macroscopic initiation stress to transformation strain would fail in a polycrystal. This is consistent with the experiments of DALY et al. [10].

Our final contribution is an exploration of the general equiaxed polycrystal using the Milton-Serkov bounds (Section 5). These bounds limit the region in which the overall stress-strain response can lie. We now discuss whether these bounds are optimal and if not how they could be improved. A natural place to examine this is the critical stress for the onset of transformation. Our bounds provide an interval for this stress ranging from the Sachs stress $w / \sqrt{\alpha^{2}+\beta^{2}}$ to $\frac{2 w}{\pi} \sqrt{\frac{1}{\alpha^{2}}+\frac{1}{\beta^{2}}}$. This sounds terribly suboptimal in light of our result in Section 3 regarding the optimality of the Sachs bound (this result would suggest that the interval collapse to a single point $w / \sqrt{\alpha^{2}+\beta^{2}}$ in an optimal bound). However, our result on the optimality of the 
Sachs bound holds only for granular polycrystals while the Milton-Serkov bounds is microstructure-independent and therefore holds for all polycrystals including those defined as sequences. For reasons that we discussed earlier in this section, it is entirely possible that microstructure-independent optimal bounds for the critical stress for the onset of transformation yield an interval. So the fact that our bound based on Milton and Serkov's approach gives us an interval does not immediately say that it is sub-optimal.

To examine the optimality, we look at the situation when $\alpha \ll \beta$. We can set $\beta=1$ without loss of generality. Then, our bounds say that critical stress for transformation is bounded from above by a quantity that is of order $1 / \alpha$. We now compare this with the work of KoHN and LiTTLE [16]. They examined the critical stress for yield of a polycrystal made of a rigid/perfectly-plastic material with two orthogonal slip systems with critical stresses $M$ and 1 with $M \gg 1$. In this situation, the mesoscale energy is homogeneous of degree one with a corner at the origin in the shape of an inverted pyramid with four sides, two with slope 1 and two with slope $M$. In short, the energy is similar near the origin to our situation with $M$ identified with $1 / \alpha$, compare Fig. 7 . They showed that as $M$ becomes large, the critical stress for yield is bounded from above by a quantity of order $\sqrt{M}{ }^{2}$ It follows that the critical stress in our transformation should be bounded from above by $1 / \sqrt{\alpha}$ and this is a significant improvement over what we have, $1 / \alpha$.

One can analyze the extent of transformation and the set of recoverable strains analogously. In the situation where $\alpha \ll \beta=1$, our bounds show that the maximum recoverable strain is bounded by a quantity of order $\alpha$ while arguments of KoHn and NieThammer [17] can be adapted to show that they are in fact bounded by a quantity of order $\alpha^{2}$. Thus, our bounds are clearly sub-optimal.

The Milton-Serkov is a microstructure-independent bound that considers only one direction (that is, one $\bar{e}$ and one $\bar{s}$ ). Thus, the bounds have to hold even for exceptional microstructures like laminates in series/parallel which are extremely anisotropic. Instead, the Kohn-Little and Kohn-Niethammer bounds consider multiple directions simultaneously and thus hold for isotropic materials. It is entirely possible to combine the two approaches. However this adds significantly to the calculations and is thus a goal for the future.

Acknowledgements. We are delighted to acknowledge various enlightening and enjoyable discussions with Pierre Suquet and Christian Lexcellent during the course of this work. This work started when ANJA SCHLÖMERKEMPER held a postdoctoral position at the University of Oxford and was continued while ANJA SCHLÖMERKEMPER was affiliated to the Institute for Analysis, Dynamics and Modeling at the University of Stuttgart. The work was completed during KAUSHIK BHATTACHARYA's sabbatical leave of absence at the Cavendish Laboratory and Clare Hall of Cambridge. We gratefully acknowledge the financial support of the EU TMR Network (FMRX-CT 98-0229, AS), the US Department of Energy (PSAAP, $\mathrm{KB})$, UK EPSRC (KB).

\footnotetext{
${ }^{2}$ We note that Goldzstein [13] showed that this $\sqrt{M}$ bound is optimal in the sense of scaling.
} 
Open Access This article is distributed under the terms of the Creative Commons Attribution Noncommercial License which permits any noncommercial use, distribution, and reproduction in any medium, provided the original author(s) and source are credited.

\section{References}

1. Ball, J.M., James, R.D.: Proposed experimental tests of a theory of fine microstructure, and the two-well problem. Philos. Trans. R. Soc. Lond. A 338, 389-450 (1992)

2. Bhattacharya, K.: Microstructure of martensite. Oxford Series of Materials Modelling. Oxford University Press, New York, 2003

3. Bhattacharya, K., Kohn, R.V.: Symmetry, texture and the recoverable strain of shapememory polycrystals. Acta Mater. 44, 529-542 (1996)

4. Bhattacharya, K., Kohn, R.V.: Elastic energy minimization and the recoverable strains of polycrystalline shape-memory materials. Arch. Rational Mech. Anal. 139, 99-180 (1997)

5. Bhattacharya, K., Schlömerkemper, A.: Transformation yield surface of shapememory alloys. J. Phys. IV France 115, 155-162 (2004)

6. Bhattacharya, K., Schlömerkemper, A.: On the Sachs bound in stress-induced phase transformations in polycrystalline scalar shape-memory alloys. PAMM Proc. Appl. Math. Mech. 8, 10569-10570 (2009)

7. Bhattacharya, K., Suquet, P.: A model problem concerning recoverable strains of shape-memory polycrystals. Proc. R. Soc. Lond. A 461, 2797-2816 (2005)

8. Brenner, R., Lebensohn, R.L., Castelnau, O.: Elastic anisotropy and yield surface estimates of polycrystals. Int. J. Solids Struct. 46(16), 3018-3026 (2009)

9. BRInSON, L.C., SCHMidT, I., LAMmERING, R.: Stress-induced transformation behavior of a polycrystalline NiTi shape memory alloy: micro and macromechanical investigations via in situ optical microscopy. J. Mech. Phys. Solids 52, 1549-1571 (2004)

10. Daly, S., Ravichandran, G., Bhattacharya, K.: Stress-induced martensitic phase transformation in thin sheets of Nitinol. Acta Mater. 55, 3593-3600 (2007)

11. Dacorogna, B.: Direct Methods in the Calculus of Variations. Springer, New York, 1989

12. DeSimone, A., James, R.D.: A constrained theory of magnetoelasticity. J. Mech. Phys. Solids 50, 283-320 (2002)

13. Goldsztein, G.H.: Rigid perfectly plastic two-dimensional polycrystals. Proc. R. Soc. Lond. A 457, 2789-2798 (2001)

14. Hutchinson, J.W.: Elastic-plastic behavior of polycrystalline metals and composites. Proc. R. Soc. Lond. A 319, 247-272 (1970)

15. James, R.D.: Displacive phase transformations in solids. J. Mech. Phys. Solids 34, 359-394 (1986)

16. Kohn, R.V., LitTLe, T.D.: Some model problems of polycrystal plasticity with deficient basic crystals. SIAM J. Appl. Math. 59, 172-197 (1998)

17. Kohn, R.V., Niethammer, B.: Geometrically nonlinear shape-memory polycrystals made from a two-variant material. ESIAM: Math. Model. Num. Anal. 2, 377-398 (2000)

18. Lexcellent, C., Vivet, A., Bouvet, C., Calloch, S., Blanc, P.: Experimental and numerical determinations of the initial surface of phase transformation under biaxial loading in some polycrystalline shape-memory alloys. J. Mech. Phys. Solids 50, 27172735 (2002)

19. Lexcellent, C., Schlömerkemper, A.: Comparison of several models of the determination of the phase transformation yield surface in shape-memory alloys with experimental data. Acta Mater. 55, 2995-3006 (2007)

20. Milton, G.W., Serkov, S.K.: Bounding the current in nonlinear conduction composites. J. Mech. Phys. Solids 48, 1295-1324 (2000) 
21. Schlömerkemper, A.: On a Sachs bound for stress-induced phase transformations in polycrystalline shape memory alloys. PAMM Proc. Appl. Math. Mech. 6, 507-508 (2006)

22. SHIELD, T.W.: Orientation dependence of the pseudoelastic behavior of single-crystals of Cu-Al-Ni in tension. J. Mech. Phys. Solids 43, 869-895 (1995)

23. Shu, Y.C., Bhattacharya, K.: The influence of texture on the shape-memory effect in polycrystals. Acta Mater. 46, 5457-5473 (1998)

24. ŠITTNER, P., NovÁK, V.: Anisotropy of martensitic transformations in modeling of shape memory alloy polycrystals. Int. J. Plast. 16, 1243-1268 (2000)

25. Zhang, Z., James, R.D., Müller, S.: Energy barriers and hysteresis in martensitic phase transformations. Acta Materialia 57(15), 4332-4352 (2009)

Division of Engineering and Applied Science,

California Institute of Technology, Pasadena, CA 91125, USA.

e-mail: bhatta@caltech.edu

and

Max Planck Institute for Mathematics in the Sciences, Inselstraße 22-26, 04103 Leipzig, Germany. e-mail: schloem@mis.mpg.de

(Received April 14, 2009 / Accepted June 15, 2009)

Published online October 22, 2009 - (c) The Author(s) (2009)

This article is published with open access at Springerlink.com 